Description of ALARMA: the alarm algorithm developed for the Nuclear Car Wash

T. Luu, P. Biltoft, J. Church, M.-A. Descalle, J. Hall, D. Manatt, J. Mauger, E. Norman, D. Petersen, J. Pruet, S. Prussin, D. Slaughter

January 26, 2007 
This document was prepared as an account of work sponsored by an agency of the United States Government. Neither the United States Government nor the University of California nor any of their employees, makes any warranty, express or implied, or assumes any legal liability or responsibility for the accuracy, completeness, or usefulness of any information, apparatus, product, or process disclosed, or represents that its use would not infringe privately owned rights. Reference herein to any specific commercial product, process, or service by trade name, trademark, manufacturer, or otherwise, does not necessarily constitute or imply its endorsement, recommendation, or favoring by the United States Government or the University of California. The views and opinions of authors expressed herein do not necessarily state or reflect those of the United States Government or the University of California, and shall not be used for advertising or product endorsement purposes.

This work was performed under the auspices of the U.S. Department of Energy by University of California, Lawrence Livermore National Laboratory under Contract W-7405-Eng-48. 


\section{Description of ALARMA: the alarm algorithm developed for the Nuclear Car Wash Thomas Luu}

P. Biltoft, J.A. Church, M.-A. Descalle, J. Hall, D. Manatt, J. Mauger, E.B.

Norman, D. Petersen, J. Pruet, S. Prussin, D.R. Slaughter

November 28, 2006 


\section{Preface}

This work was performed under the auspices of the U.S. Department of Energy by the University of California, Lawrence Livermore National Laboratory under contract No. W-7405-Eng-48. 


\section{Contents}

1 Introduction $\quad 9$

2 Methodology 11

2.1 Experimental Data in 2 Dimensions . . . . . . . . . . . . 11

2.2 Shape Independence of Spectrum . . . . . . . . . . . . . 18

2.3 Fitting 2-D Data . . . . . . . . . . . . . . . . . . 23

2.4 Why Not Fit to Decay Constants? . . . . . . . . . . . . . 25

2.5 Construction of Basis Functions . . . . . . . . . . . . . 25

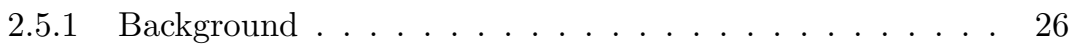

2.5.2 HEU . . . . . . . . . . . . . . . . . . . . 27

2.5.3 ${ }^{16} \mathrm{~N}$ interference . . . . . . . . . . . . . . . . 27

2.5.4 Other interferences . . . . . . . . . . . . . . 27

2.6 Importance of Dead-time Corrections . . . . . . . . . . . . . . . 32

2.7 Constructing Receiver Operator Characteristic (ROC) Curves . 33

2.7.1 Why bootstrap the data? . . . . . . . . . . 34

3 Data Analysis $\quad 37$

3.1 Cargoes with HEU . . . . . . . . . . . . . . . . . 37

3.2 Samples with Teflon Contamination . . . . . . . . . . 37

3.3 Cargoes without HEU . . . . . . . . . . . . . . . . . 39

3.4 ROC curves, false alarm rates, and optimal fitting windows . . . 44

3.4.1 Optimal time window . . . . . . . . . . . . . . 45

3.4 .2 Optimal energy window . . . . . . . . . . . . . 47

3.5 Dependence on temperature fluctuations . . . . . . . . . . . . 49

4 Coding Logic and Procedures $\quad 51$

4.1 calibrateAlarma . . . . . . . . . . . . . . 51

4.2 basisAlarma . . . . . . . . . . . . . . 54

4.3 fitAlarma...................... 54

5 Conclusion $\quad 57$

5.1 Things to be done $\ldots \ldots \ldots \ldots \ldots \ldots$ 
CONTENTS 


\section{List of Figures}

2.1 Experimental data showing active wood cargo . . . . . . . . . 12

2.2 Experimental data showing active steel cargo . . . . . . . . . . . 13

2.3 Data with average background subtracted . . . . . . . . . . . . 14

2.4 Sample HEU in wood and ${ }^{16} N$ signatures. . . . . . . . . . . . . 15

2.5 Difference between active background and HEU signatures in wood. 16

2.6 Comparison of HEU signature from wood and steel cargoes. . . . 17

2.7 Differential cross section for Compton scattering. . . . . . . . . . 19

2.8 Klein-Nishina cross section . . . . . . . . . . . . . 20

2.9 Probabilities for final photon energies below various cutoff energies due to Compton scattering. . . . . . . . . . . . . . . . 21

2.10 Monte Carlo photon transport configuration. . . . . . . . . . . . 21

2.11 Spectrum of photons traversing empty container. . . . . . . . . 22

2.12 Attenuation for wood and aluminum cargoes . . . . . . . . . . 22

2.13 Attenuation for steel inhomogeneous cargoes . . . . . . . . . . 23

2.14 Schematic diagram of Eq. 2.4. . . . . . . . . . . . . . 24

2.15 Example of wood active background data and basis function . . . 28

2.16 Example of steel active background data and basis function . . . 29

2.17 Example of wood active background data and basis function . . . 30

2.18 Sample ${ }^{16} \mathrm{~N}$ basis function. . . . . . . . . . . . . . . . . . . . 31

2.19 Energy-counts profile at various times . . . . . . . . . . . . 32

2.20 Summed counts above $8 \mathrm{MeV}$. . . . . . . . . . . . . 34

3.1 Sample HEU fit . . . . . . . . . . . . . . . . . . . . . . . 38

3.2 Sample HEU fit distribution . . . . . . . . . . . . . . . . . . . 39

3.3 Various HEU fit distributions for wood and steel . . . . . . . . . 40

3.4 Fit performed with HEU and teflon in wood . . . . . . . . . . . . 41

3.5 Fit performed with HEU and teflon in steel . . . . . . . . . . . . 42

3.6 Null distribution . . . . . . . . . . . . . . . . . . . . . 43

3.7 Overlap of distributions . . . . . . . . . . . . . . . . . . . . . . . . . . . . 44

3.8 ROC curves calculated from Fig. 3.7 . . . . . . . . . . . . . . . . 45

3.9 ROC curves for various time windows . . . . . . . . . . . . 46

3.10 Signal-to-background ratio as a function of time window . . . . . 46

3.11 Plot of $\chi^{2} /$ d.o.f. . . . . . . . . . . . . . . . . . 47

3.12 Signal-to-background ratio as a function of lower energy cutoff . 48 
3.13 Dependence of distributions on temperature . . . . . . . . . . . 49

3.14 Dependence of ROC curves on temperature . . . . . . . . . . 50

4.1 Sample calibration runs. . . . . . . . . . . . . . . . 52

4.2 Sample calibration using a linear fitting function. . . . . . . . . 53

4.3 Sample calibration using a quadratic fitting function. . . . . . . . 53 


\section{List of Tables}

2.1 Features of representation of data in energy-time domain . . . . 25

2.2 Savitzky-Golay fitting parameters . . . . . . . . . . . . 26

2.3 Sample deadtime errors at various times . . . . . . . . . . . 33

4.1 Calibration peaks for Yttrium and Nitrogen . . . . . . . . . . 52 


\section{Chapter 1}

\section{Introduction}

The goal of any alarm algorithm should be that it provide the necessary tools to derive confidence limits on whether the existence of fissile materials is present in cargo containers. It should be able to extract these limits from (usually) noisy and/or weak data while maintaining a false alarm rate (FAR) that is economically suitable for port operations. It should also be able to perform its analysis within a reasonably short amount of time (i.e. $\approx$ seconds). To achieve this, it is essential that the algorithm be able to identify and subtract any interference signature that might otherwise be confused with a fissile signature. Lastly, the algorithm itself should be user-intuitive and user-friendly so that port operators with little or no experience with detection algorithms may use it with relative ease. In support of the Nuclear Car Wash project at Lawrence Livermore Laboratory, we have developed an alarm algorithm that satisfies the above requirements. The description of the this alarm algorithm, dubbed ALARMA, is the purpose of this technical report.

The experimental setup of the nuclear car wash has been well documented[1, $2,3]$. The presence of fissile materials is inferred by examining the $\beta$-delayed gamma spectrum induced after a brief neutron irradiation of cargo, particularly in the high-energy region above approximately $2.5 \mathrm{MeV}$. In this region naturally occurring gamma rays are virtually non-existent. Thermal-neutron induced fission of ${ }^{235} U$ and ${ }^{239} P$, on the other hand, leaves a unique $\beta$-delayed spectrum[4]. This spectrum comes from decays of fission products having half-lives as large as 30 seconds, many of which have high Q-values. Since high-energy photons penetrate matter more freely, it is natural to look for unique fissile signatures in this energy region after neutron irradiation. The goal of this interrogation procedure is a $95 \%$ success rate of detection of as little as 5 kilograms of fissile material while retaining at most $.1 \%$ false alarm rate.

Plywood is used to simulate hydrogenous cargo material and steel (pipes) is used to simulate metallic cargo. The wood consists of $120 \times 240 \mathrm{~cm}$ sheets and has approximately $.65 \mathrm{~g} / \mathrm{cm}^{3}$. The steel pipes have approximately $10 \mathrm{~cm}$ diameters $\times 6.4 \mathrm{~mm}$ wall thickness are $.6 \mathrm{~g} / \mathrm{cm}^{3}$. Fissile sources consist of a 'large' (380 g) and 'small' (250 g) sample of HEU ( $U_{3} O_{8} 94 \%$ enriched). Note 
that the masses of the HEU sources used in our experimental runs are at least an order of magnitude smaller than 5 kilograms. Runs are done with either wood or steel cargoes stacked at various heights and the HEU sources placed at various depths within the cargo. 


\section{Chapter 2}

\section{Methodology}

In this chapter we motivate the reason for developing an alarm algorithm that utilizes the full energy-time domain of the experimental data. An essential assumption of our fitting routine is the near independence of the high-energy spectra on the type of cargo media. This assumption, which we justify below, greatly simplifies the analysis of data since the number of basis functions used by our fitting routines is kept to a minimum. We also discuss the construction of these basis functions, as well as enumerate our reasons for moving away from an algorithm that fits summed spectra to decay curves. We note that our analysis so far has been performed on static cargo (i.e. cargo was not moving during irradiation and scanning).

\subsection{Experimental Data in 2 Dimensions}

Figures 2.1 and 2.2 show example data sets obtained from experimental runs in which wood and steel cargoes were irradiated by a neutron beam (with endpoint energy $7 \mathrm{MeV}$ ) for 30 seconds. The data shows the subsequent energy spectrum up to 98 seconds after the irradiation was turned off. The $\mathrm{x}$ - and $\mathrm{y}$-axes display the time and energy binning of the data, respectively, while the z-axis displays the counts binned at one-second intervals. In Fig. 2.3 we show the corresponding close-ups of Figs. 2.1 and 2.2 where we have subtracted from each energy bin the average of the data in the final 10 seconds. Plot (a) shows the active wood example while (b) shows the active steel results. Note that in both cases the spectrum is essentially flat in the high-energy regime for all times. At lower energies (which is not shown) the spectrum is formed by in-medium activation of the cargo and interactions of the detector with cosmic ray particles.

In Fig. 2.4 we show an example where HEU (a) and separately teflon (b) is placed within the wood cargo. The latter simulates an ${ }^{16} \mathrm{~N}$ interference signal. In both cases the average energy calculated from the final 10 seconds has also been subtracted. It is obvious that the spectrum is not flat in the high-energy region above approximately $2.0 \mathrm{MeV}$. 


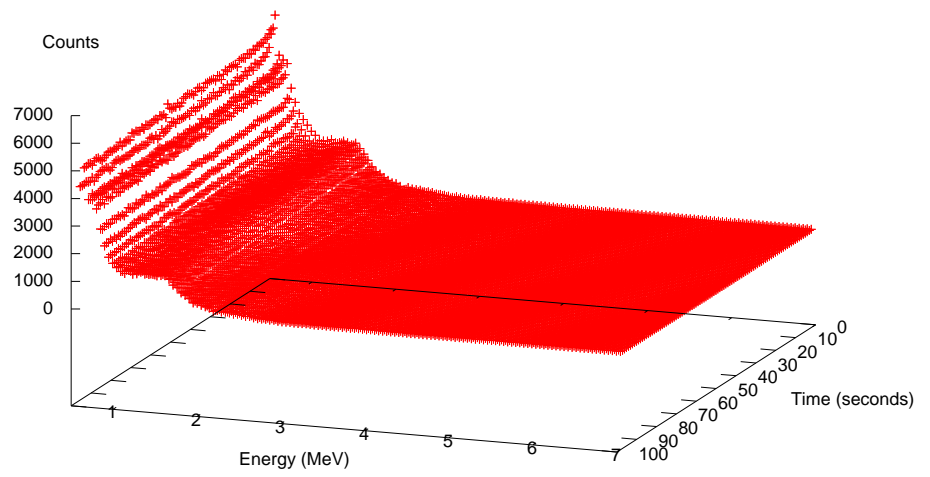

(a)

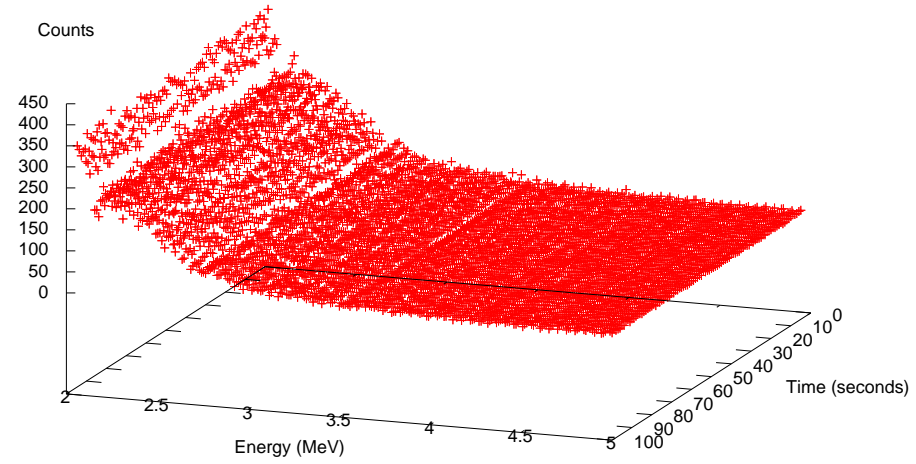

(b)

Figure 2.1: Experimental data residing in full time-energy-counts space. Plot (a) shows the results for wood active background. Plot (b) shows a close up in the 2 to $5 \mathrm{MeV}$ range. Dataset:[20060718_run015] 


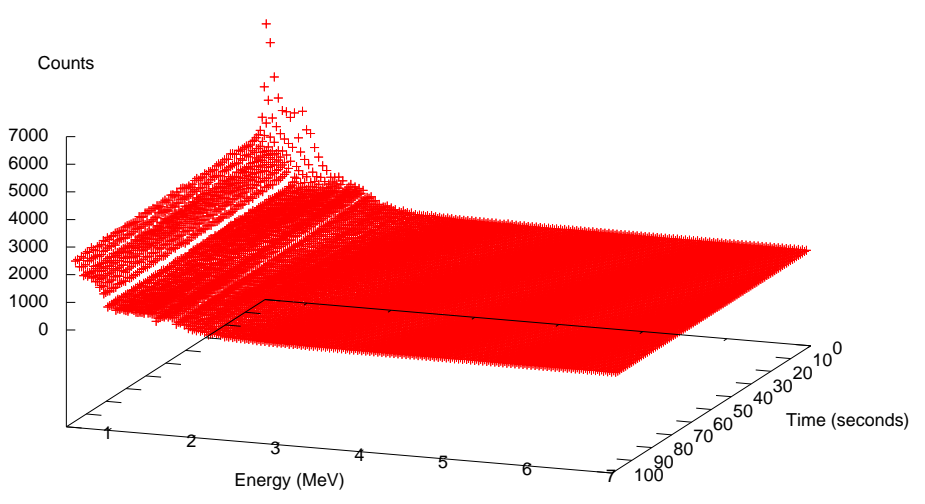

(a)

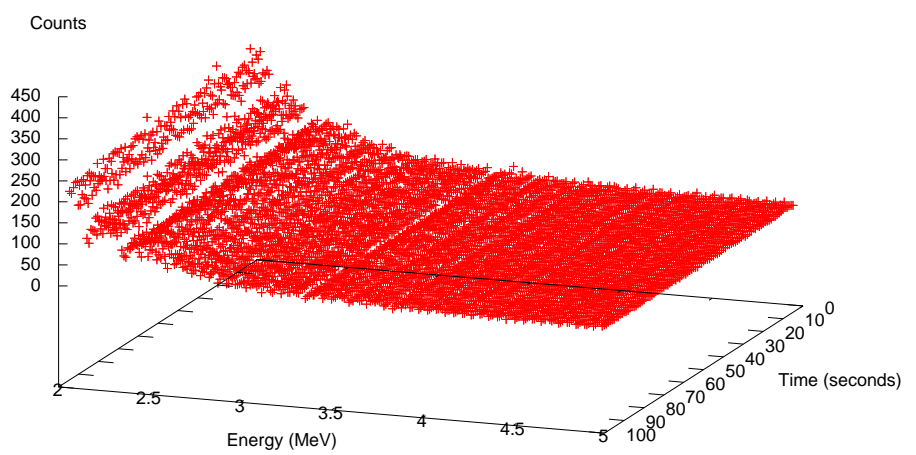

(b)

Figure 2.2: Experimental data residing in full time-energy-counts space. Plot (a) shows the results for steel active background. Plot (b) shows a close up in the 2 to $5 \mathrm{MeV}$ range. Data set:[20060810_run016] 


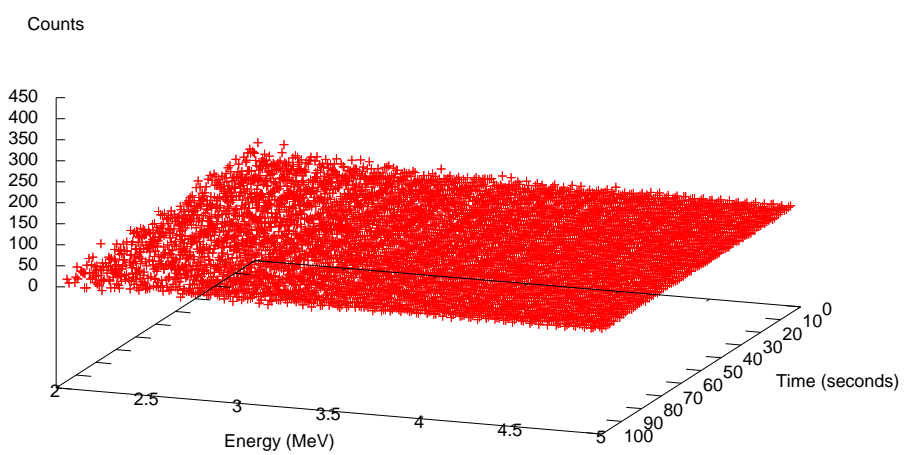

(a)

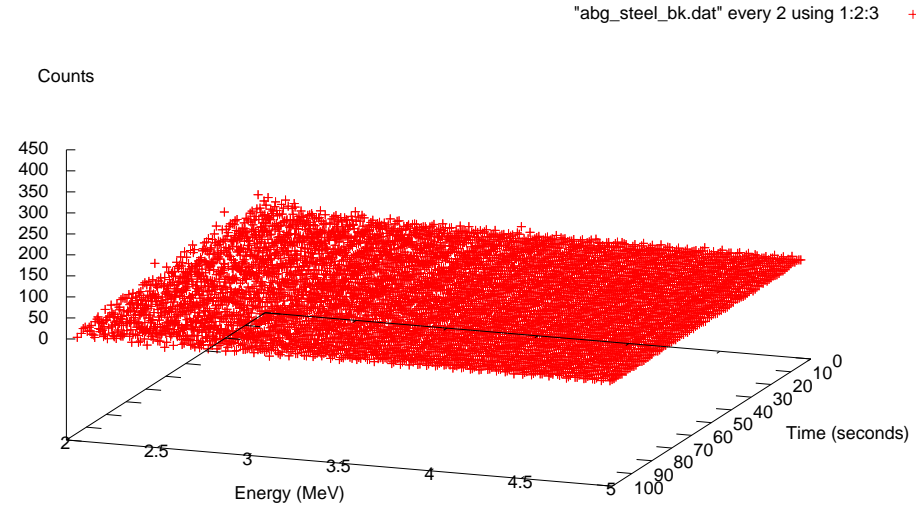

(b)

Figure 2.3: Wood (a) and steel (b) active backgrounds where the average of the final ten seconds for each energy bin was subtracted. Data sets:[20060810_run016, 20060718_run015] 


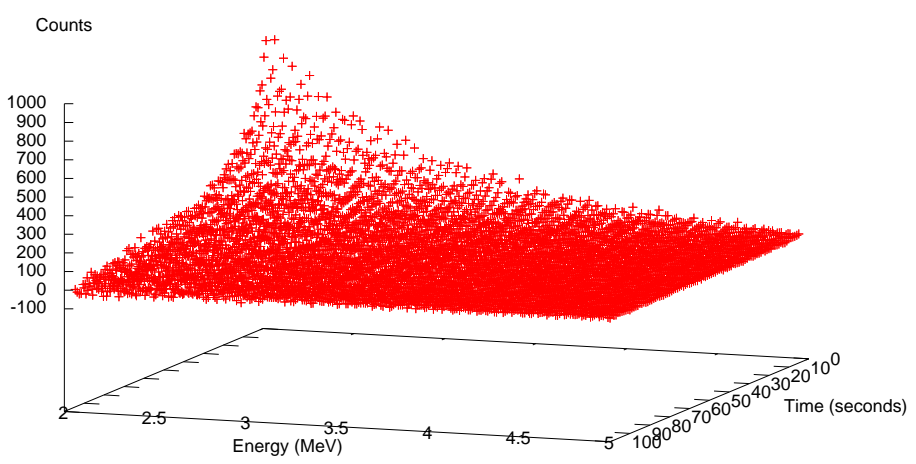

(a)

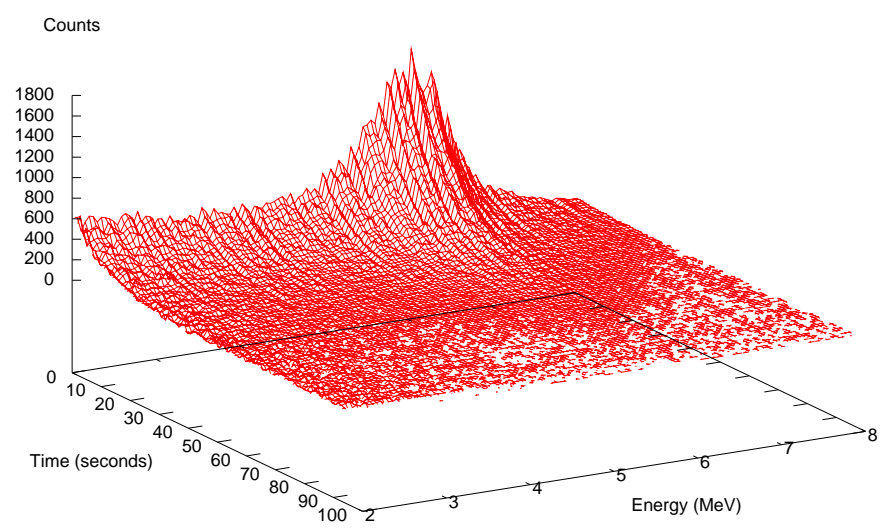

(b)

Figure 2.4: Plot (a) shows the results for HEU imbedded in wood. Plot (b) shows the spectrum due to the presence of ${ }^{16} N$. Data sets:[20060711_run007, 20060718_run005] 


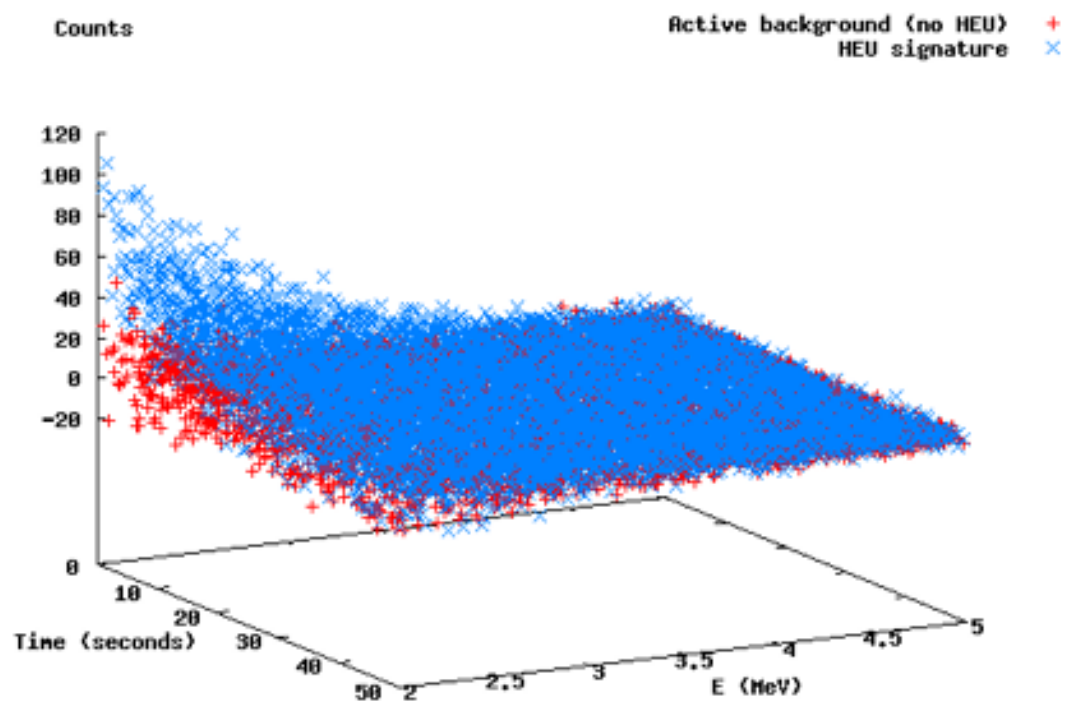

Figure 2.5: Signatures of active background (red) and HEU in wood (blue) are overlaid to show differences. Both experiments consisted of cargo with 4 feet of wood (data sets). Spectrum is shown only above $2 \mathrm{MeV}$.

The difference in the signatures above $2.0 \mathrm{MeV}$ of an active background spectrum and a spectrum in which HEU is present can be quite pronounced, even for weak HEU signatures. Fig. 2.5 overlays two such signatures to show this difference. Here the spectrums are shown above $2 \mathrm{MeV}$ and the cargo consists of 4 feet of wood. The HEU signature is very weak for this example, yet its signature (blue) is visually distinct from the active wood background (red) in this energy range.

On the other hand, the HEU signatures within different cargoes are very similar in shape, as Fig. 2.6 shows. Here the HEU signature is shown from cargoes of wood and steel. Aside from an overall normalization (i.e. different total counts), the shape of the spectrum is very similar in the high-energy region. This is true for spectrums due to other sources, such as ${ }^{16} \mathrm{~N}$. The relative independence of the spectrum shape of HEU from different cargoes above $2 \mathrm{MeV}$ is an essential feature utilized by our alarm algorithm's fitting routines. As will be explained later (Sect. 2.3), this independence greatly facilitates the analysis of data from various irradiated cargo media, since only one basis function for each source will be needed and can be used for all the different types of cargoes 


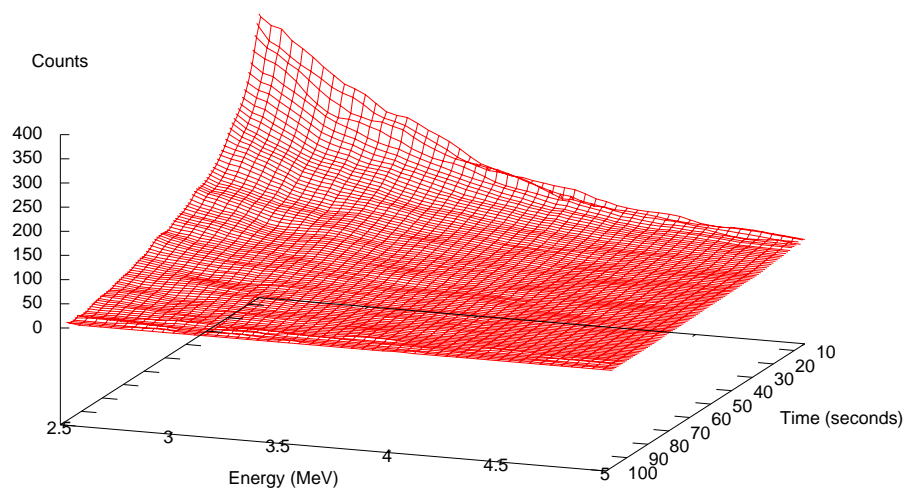

(a)

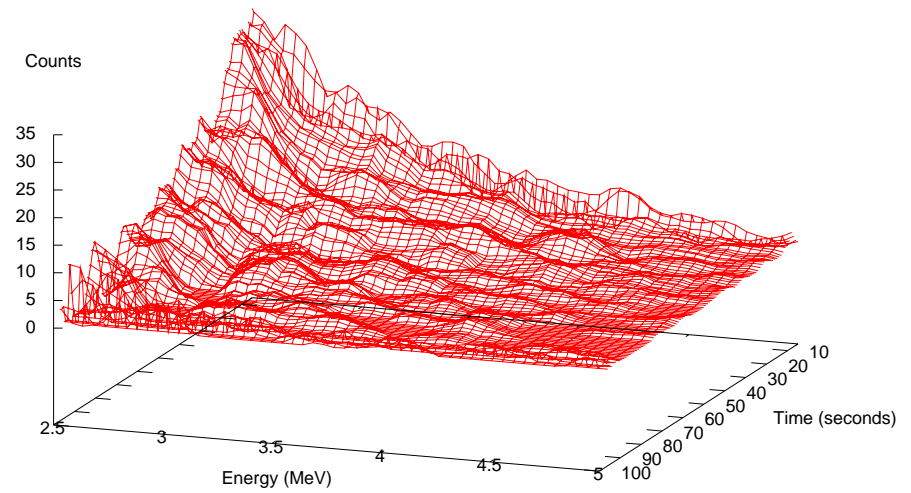

(b)

Figure 2.6: HEU signatures originating from wood cargo (a) and steel cargo (b). Aside from an overall normalization, the spectrum is essentially the same above approximately $2.5 \mathrm{MeV}$. The data has been smoothed for ease of viewing. 
in the high-energy region. Before describing our fitting routines, however, it is prudent to understand physically why this independence is manifest. The next section addresses this issue.

\subsection{Shape Independence of Spectrum}

The dominant process in which $\gamma$-rays interact with cargo media in the $2-10 \mathrm{MeV}$ energy range is via Compton scattering, where the initial photon scatters off of a (essentially) free electron. At or above $10 \mathrm{MeV}$, pair production, where the photon is converted to electron/positron pairs, becomes the dominant process. In principle, pair production can occur below $10 \mathrm{MeV}$ and above $1.06 \mathrm{MeV}$ (twice the electron mass), but due to kinematical constraints (e.g. conservation of momentum) this process is suppressed compared to Compton scattering.

The differential cross section due to Compton scattering of unpolarized photons off electrons (and more generally, point-like spin- $1 / 2$ particles) is given by the Klein-Nishina formula[5],

$$
\frac{d \sigma}{d \Omega}=\frac{1}{2}\left(\frac{e^{2}}{m_{e}}\right)^{2}\left[\frac{\omega_{f}}{\omega_{i}}-\left(\frac{\omega_{f}}{\omega_{i}}\right)^{2} \sin ^{2}(\theta)+\left(\frac{\omega_{f}}{\omega_{i}}\right)^{3}\right],
$$

where $m_{e}$ is the electron mass, $\omega_{i}$ and $\omega_{f}$ are the initial and final energies, respectively, of the photon (we have assumed natural units, $c=1, \hbar=1$ ), $\theta$ is the angle relative to the incoming photon's direction, and

$$
\frac{\omega_{f}}{\omega_{i}}=\frac{1}{1+\frac{\omega_{i}}{m_{e}}(1-\cos (\theta))}
$$

Note that $e^{2} / m_{e}=2.82 \times 10^{-13} \mathrm{~cm}$ is the classical electron radius. Figure 2.7 plots this function for initial photon energies of $2,3,4$, and $5 \mathrm{MeV}$. Notice that the differential cross section is strongly peaked in the forward direction for photons at these energies. The total cross section is simply

$$
\sigma(\nu)=\left(\frac{e^{2}}{m_{e}}\right)^{2}\left[\frac{2 \pi(2+\nu(1+\nu)(8+\nu))}{\nu^{2}(1+2 \nu)^{2}}-\frac{\pi(2+\nu(\nu-2)) \log (1+2 \nu)}{\nu^{3}}\right],
$$

where $\nu=\omega_{i} / m_{e}$. Figure 2.8 plots this cross section for photon energies up to 8 $\mathrm{MeV}$. Notice that above $\approx 2.5 \mathrm{MeV}$, the variation in the cross section is small.

Prussin has investigated the likelihood that given a photon with initial energy $\omega_{i}$ that undergoes Compton scattering, the final energy $\omega_{f}$ of the photon is below some cutoff energy $\omega_{c}$. This probability, $P\left(\omega_{f} \leq \omega_{c}: \omega_{i}\right)$, is defined as

$$
P\left(\omega_{f} \leq \omega_{c}: \omega_{i}\right)=\frac{2 \pi}{\sigma\left(\omega_{i}\right)} \int_{-1}^{x_{c}} d(\cos (\theta)) \frac{d \sigma\left(\omega_{i}\right)}{d \Omega},
$$

where $d \sigma\left(\omega_{i}\right) / d \Omega$ is given by Eq. 2.1, $\sigma\left(\omega_{i}\right)$ is the total Compton cross section for initial photon energy $\omega_{i}$ (Eq. 2.2), and

$$
x_{c}=1-m_{e}\left(\frac{1}{\omega_{c}}-\frac{1}{\omega_{i}}\right) \text {. }
$$




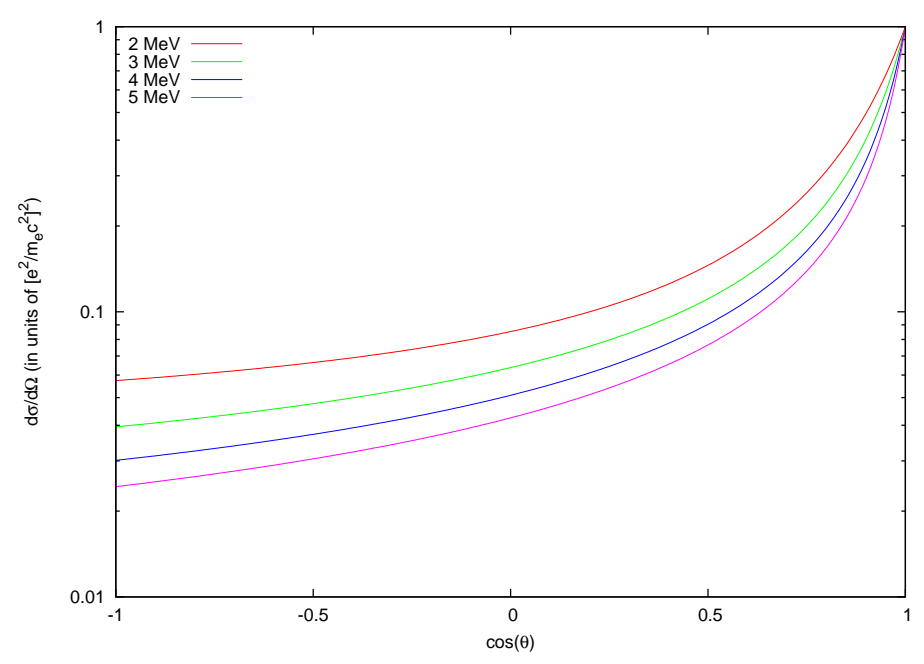

Figure 2.7: Differential cross section for Compton scattering of photon of energies $2,3,4$, and $5 \mathrm{MeV}$ off electron.

In Fig. 2.9 we plot this probability for various cutoff energies as a function of initial photon energy. From the figure it can be deduced that given a photon with initial energy $\omega_{i}$, if it undergoes Compton scattering, the probability that it will down-scatter below some cutoff energy $\omega_{c}<3 \mathrm{MeV}$ is large. In most cases this probability is greater than $70 \%$. Thus this analysis shows that, after traversing through media, a detected photon of energy greater than $\approx 3 \mathrm{MeV}$ most likely did not interact with the cargo media, since if it did scatter it would very likely have scattered to an energy below this threshold. This analysis is valid only in the Compton (incoherent) limit.

It is stressed that the probability defined above does not reflect the likelihood that a photon undergoes Compton scattering. It only states that once a photon undergoes Compton scattering, the probability that the photon loses much of its initial energy is large. The likelihood that a photon undergoes Compton scattering depends linearly, to first order, on the density of electrons in which the photon traverses through. As such, higher $\mathrm{Z}$ elements will produce more scattering. Yet the statements of the previous paragraph do not change. Thus one can posit that the shape of the high energy spectrum of photons $(\gtrsim 2.0 \mathrm{MeV})$ should look very similar for various cargo media; only the overall normalization, or attenuation, is different.

To support this statement, we show attenuation results calculated from Monte Carlo photon transport simulations using various cargo media performed 


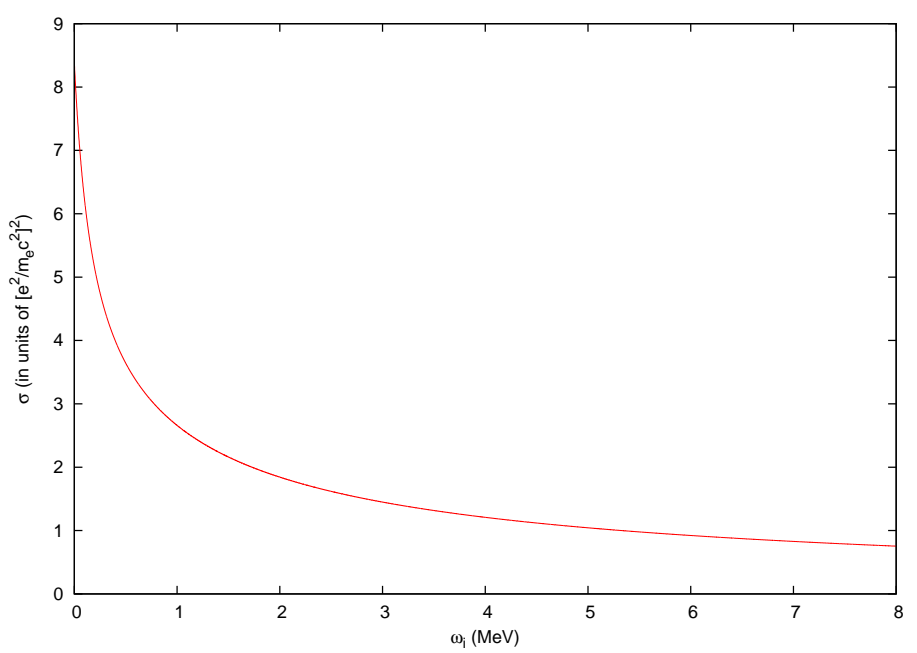

Figure 2.8: Total Compton cross section off electron as a function of incoming photon energy $\omega_{i}$.

by Hall. The configurations for the simulations are shown in Fig. 2.10. In plot (a) the cargo medium (brown) consists of either wood, aluminum, or steel. A SNM source (red), a $1 \mathrm{~kg}$ Oralloy sphere, is located at the center of the box, and two $2^{\prime} \times 4^{\prime} \times 6^{\prime \prime}$ plastic scintillator detectors (blue) are located to the sides of the cargo. The size of the cargo container for each medium is varied (or equivalently, the density of the media is varied) such that there are 0.5, 1.0, 2.0, and 4.0 equivalent mean free paths $(\mathrm{mfp})$ for photons traversing directly from the SNM to the wall of the container in line of sight of the detector.

A fiducial run was done where there was no cargo present (photons traversed through vacuum). Photons that impinged upon the detectors in this case underwent no scattering. The energy spectrum of these photons is shown in Fig. 2.11, binned in $50 \mathrm{keV}$ intervals for the energy range 2.5-6.0 MeV. For each simulation where cargo media was present the attenuation was calculated by dividing the obtained energy spectrum of photons impinging the detectors by the fiducial spectrum shown in Fig. 2.11. In Figs. 2.12 and 2.13(a) we show the attenuation as a function of photon energy for wood, aluminum and steel cargoes at various densities corresponding to different photon mean free paths. The attenuation, as a function of photon energy, is nearly constant for all three cases. The slight positive slope, most evident in wood cargo, is attributed to the hardening of the photon spectrum since the cross section decreases slightly at higher energies (see Fig. 2.8). 


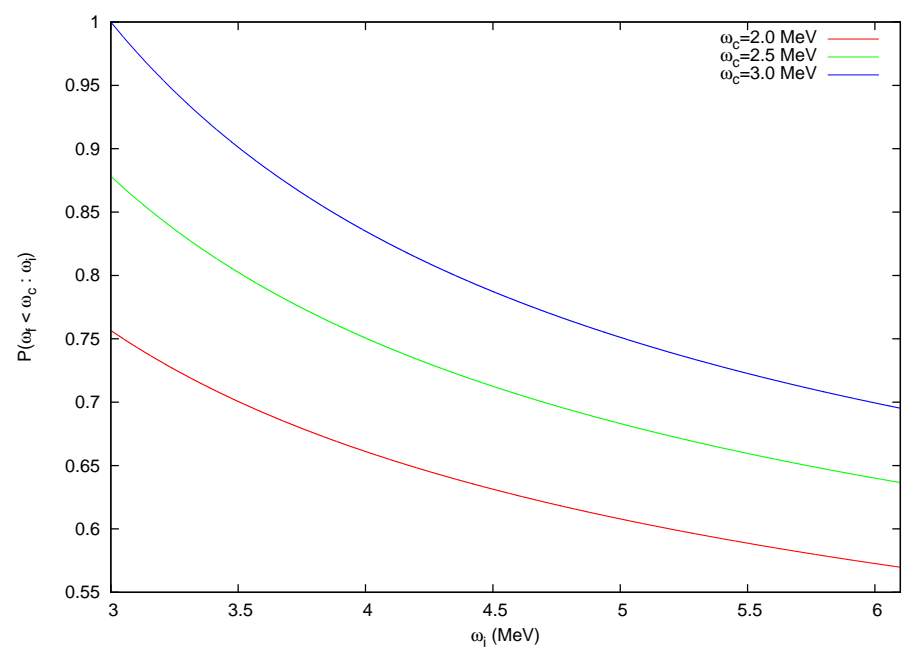

Figure 2.9: Figure shows the probability that a photon of initial energy $\omega_{i}$, after Compton scattering once, has final energy below $\omega_{c}=2.0,2.5,3.0 \mathrm{MeV}$.

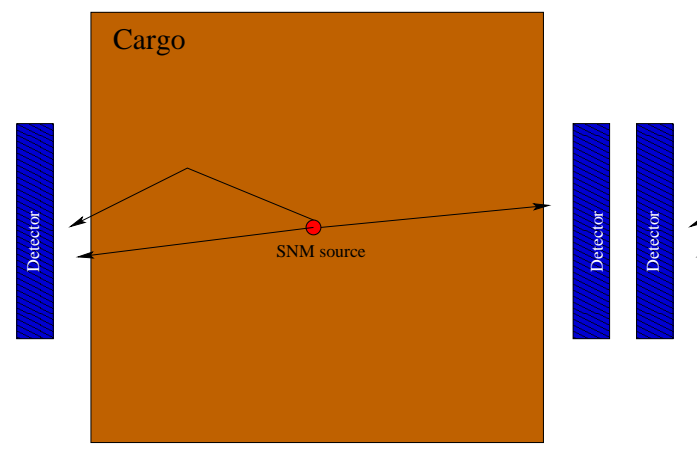

(a)

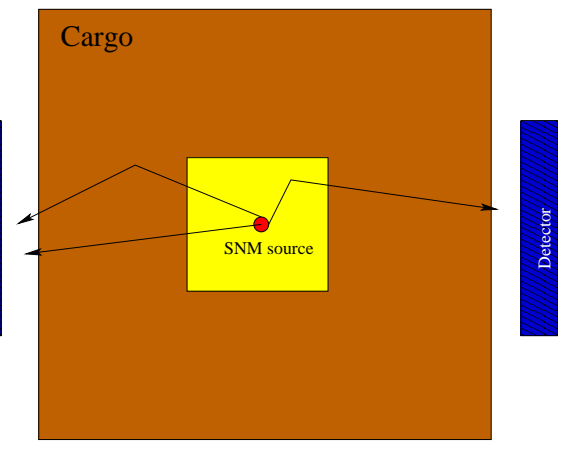

(b)

Figure 2.10: Configurations used by Hall in performing Monte Carlo photon transport simulations. Plot (a) shows a scenario of uniform cargo, whereas (b) shows a cargo configuration where the yellow square consists of steel and the brown region is wood. For both scenarios, the SNM source (red) is in the center and the two detectors (patterned blue) are located to the sides of the cargo. 


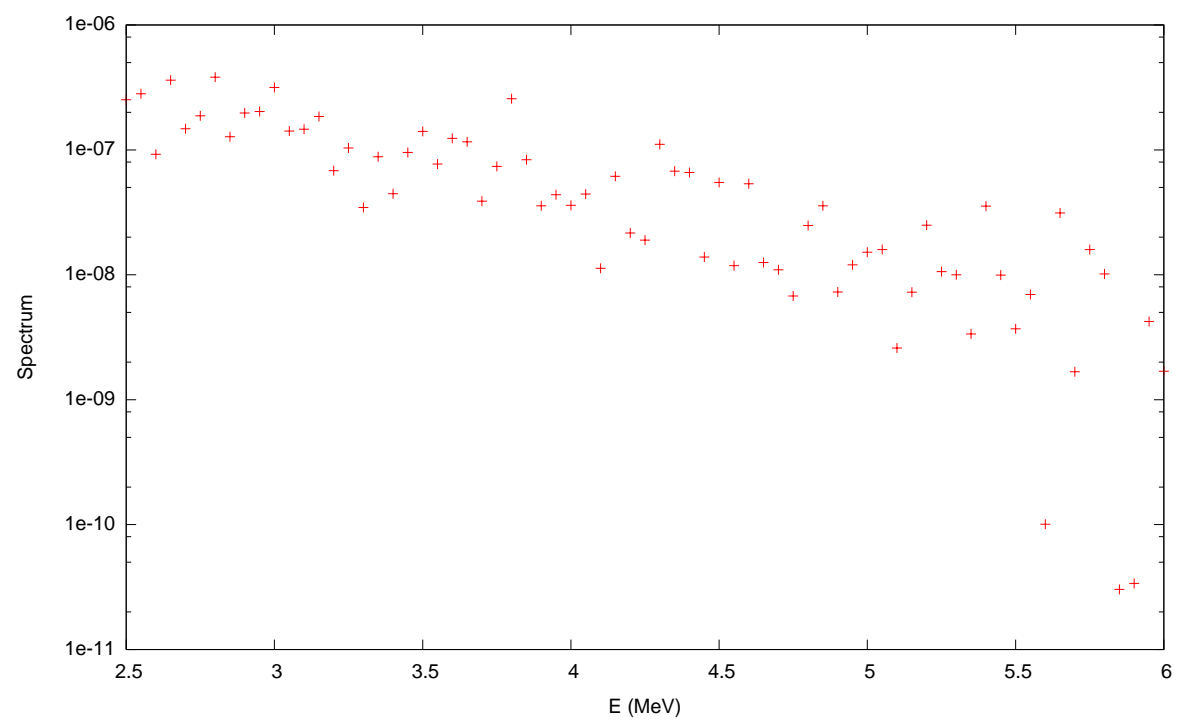

Figure 2.11: Energy spectrum of photons impinging on detectors after traversing through an empty container, as described in text and shown in Fig. 2.10(a).

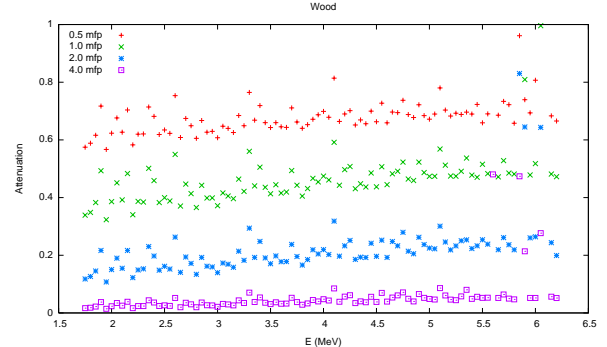

(a)

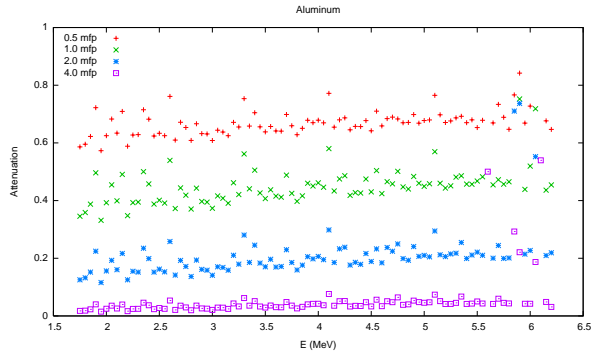

(b)

Figure 2.12: Attenuation as a function of photon energy for wood (a) and aluminum (b) cargoes at different densities corresponding to 0.5, 1.0, 2.0, and 4.0 minimal photon mfps. 


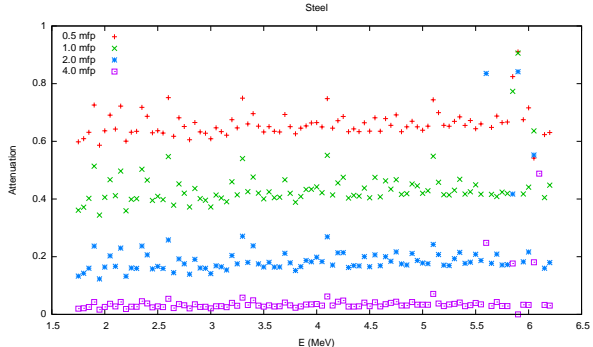

(a)

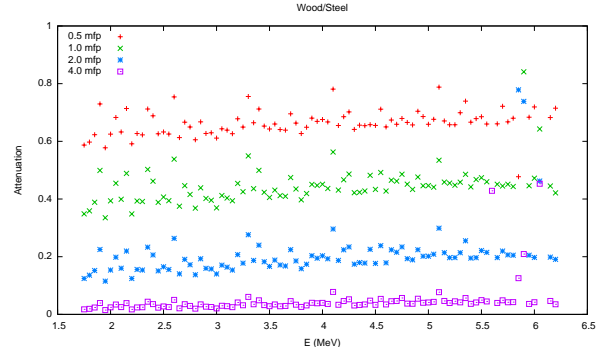

(b)

Figure 2.13: Attenuation as a function of photon energy for steel (a) and inhomogeneous cargo consisting of steel and wood (b) at different densities corresponding to $0.5,1.0,2.0$, and 4.0 minimal photon mfps.

The calculations presented above had cargo containing homogeneous media. In general, cargoes will not be homogeneous and there may be concern that attenuation in this case will not be nearly flat. In a first order attempt to address this issue, Hall has also run simulations using cargo configurations consisting of both wood and steel, as shown in Fig. 2.10(b). Here the center region surrounding the SMN source consists of steel. The entire steel box is then enclosed in wood. The densities of the wood and steel cargoes are varied such that they both have the same equivalent photon mfp in line of sight from the SNM source and detector, and that the summed mfp from both wood and steel equal 0.5, 1.0, 2.0, and 4.0 mfps. Figure 2.13(b) shows the attenuation for this configuration. Again the attenuation is nearly flat within the energy region plotted.

The near constant attenuation obtained for various cargo media indicates that the shape of the energy spectrum of photons is nearly the same for different cargo configurations. This is true for inhomogeneous as well as homogeneous media. This implies that HEU signatures obtained in wood should be the same (up to an overall constant) as HEU signatures obtained in steel, as was found experimentally in Sect. 2.1. HEU signatures obtained from cargoes containing both wood and steel (or any other combination of materials) should have the same signature as well in the high energy regime.

\subsection{Fitting 2-D Data}

To extract a fissile signature from data taken after neutron irradiation of cargo, the algorithm ALARMA attempts to fit the high-energy region of the data to a set of pre-calculated basis functions. These basis functions reside in the full 


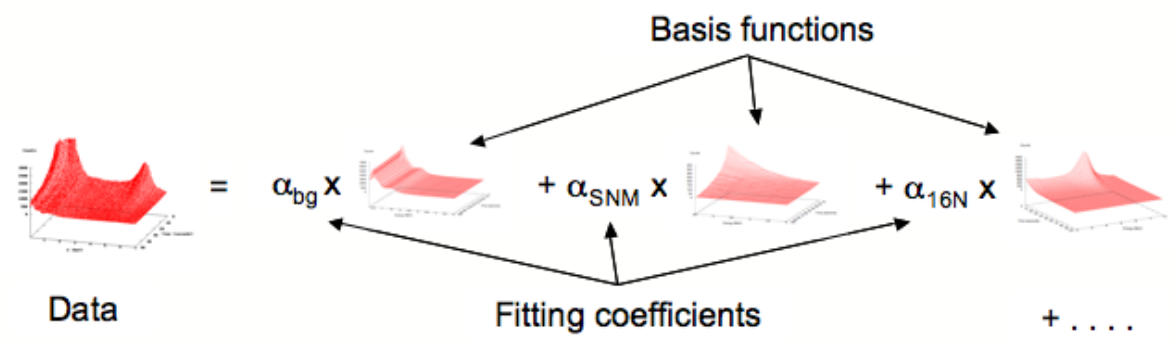

Figure 2.14: Schematic diagram of Eq. 2.4.

energy-time $(E, t)$ domain of the experimental data and represent the various components that we believe contribute to the overall spectrum. For example, there is a basis function $\Phi_{b g}(E, t)$ that represents the active background, a basis function $\Phi_{S N M}(E, t)$ that represents the fissile signature, and various other basis functions that represent possible interferences, such as $\Phi_{16}(E, t)$ for the presence of ${ }^{16} N$. In theory there will be an infinite number of interference basis functions. Fortunately, as we will describe later, practical applications so far only need one interference basis function: $\Phi_{16} \mathrm{~N}$.

The fitting algorithm will allow us to extract a set of coefficients $\alpha_{i}$ that allow us to express the experimental data in terms of the basis functions,

$$
\begin{aligned}
\Phi_{e x p}(E, t)=\alpha_{b g} \Phi_{b g}(E, t) & +\alpha_{S N M} \Phi_{S N M}(E, t) \\
& +\alpha_{16} \Phi_{16}(E, t)+\text { other interferences. . . }
\end{aligned}
$$

where $\Phi_{\exp }$ represents the experimental data. $\alpha_{S N M}>0$ would indicate that a fissile signature is present in the data, and would lead us to conclude that fissile material is present in the cargo. Notice that the basis functions have no dependence on the type of cargo (i.e. wood, steel, mixtures, etc. . .). This independence relies on the conclusions of the previous section: Up to an overall constant (i.e. $\alpha_{i}$ ), the shape of the energy spectrum at high energies is basically the same for all cargo media. We discuss the construction of these basis functions in Sect. 2.5. Figure 2.14 gives a schematic representation of Eq. 2.4. Here the $\Phi$ functions are replaced by a 3-D representation of the data.

Due to uncertainties in the experimental data and fitting routines (described in detail in Sect. 4), an extracted coefficient of $\alpha_{S N M}>0$ does not indicate the presence of fissile material with $100 \%$ confidence, nor does $\alpha_{S N M}=0$ imply that no fissile material is present with $100 \%$ confidence. The fitting coefficients themselves will have uncertainties that preclude the definite identification of whether or not fissile material is present. An analysis of these uncertainties, as well as the confidence limits and false alarm rates that can be deduced from them, is given in Sects. 2.7 and 3. 
Table 2.1: Benefits of utilizing fitting algorithm on data in original energy-time domain

\begin{tabular}{|c|}
\hline Basis function contain correct decay curves and half-lives \\
\hline Data representation is original and not manipulated \\
\hline Background easily subtracted \\
\hline Facilitates identification of interferences \\
\hline Dead-time corrections could be implemented after data-acquisition \\
\hline User-friendly 3-dimensional representation of data \\
\hline
\end{tabular}

\subsection{Why Not Fit to Decay Constants?}

An alternative to fitting basis functions in the full energy-time domain is to just sum the energy counts above some threshold (e.g. $2.5 \mathrm{MeV})$ and fit the summed counts to exponential functions. In this case, one is fitting decay curves using half-lives of the elements believed relevant to the spectrum. Indeed, preliminary analysis of the data was done in this fashion (e.g. $[3,6])$, with satisfactory results.

On the other hand, working in the original energy-time domain has additional benefits over fitting decay curves. First off, the basis functions already incorporate the correct decay curves, and thus the correct half-lives, since they are fitted from experimental data. This is explained in greater detail in Sect. 2.5. Aside from the requisite binning during data acquisition, the data is raw and not manipulated in any fashion. By viewing the data in a 3-dimensional fashion, identification of interferences, such as the ${ }^{16} \mathrm{~N}$ signature, is greatly facilitated, and subsequently easily eliminated. The background can be easily subtracted by looking at long times where any fissile signature has decayed below background levels. This eliminates a source of uncertainty and thus facilitates error analysis. If detectors are large enough, or counts sufficiently high in the 8-10 $\mathrm{MeV}$ regime, the algorithm can perform its own self dead-time corrections after data acquisition. This is explained in greater detail in Sect. 2.6 ${ }^{1}$. Lastly, by working in the energy-time domain, a 3-dimensional representation of the data can be obtained that is more user-friendly for visual inspection and identification of suspect signatures. This last feature partly addresses the point that users of this algorithm most likely will not have any technical background in nuclear physics. Table 2.1 summarizes these points succinctly.

\subsection{Construction of Basis Functions}

Originally the basis functions $\Phi$ were calculated via Monte Carlo simulations, where photons from a source were sampled and propagated to the detectors. Pruet used sources that modeled fissile materials as well as the ${ }^{16} \mathrm{~N}$ signature.

\footnotetext{
${ }^{1}$ This form of dead-time correction was not implemented in the analyses presented here.
} 
Table 2.2: Savitzky-Golay fitting parameters

\begin{tabular}{|c|c|c|}
\hline \hline $\mathrm{N}_{R}$ & $\mathrm{~N}_{L}$ & $\mathrm{M}$ \\
\hline 24 & 24 & 6 \\
\hline
\end{tabular}

Descalle simulated the energy response of the detectors, which were subsequently folded with a temporal Gaussian response function. These latter calculations simulated the interaction of the photons with the detectors. Coupling the simulated flux of photons with the response of the detector, the basis function of a particular source was constructed. In this manner, any interference basis function could be constructed through simulation as long as there was a suitable model for the source.

The biggest uncertainty of these calculations were the response functions of the detector, which were actually not known at the time of the simulations. Furthermore, transport of the photons through media was modeled naively. To address these issues we have chosen to extract the basis functions directly from experimental data of HEU signatures and ${ }^{16} \mathrm{~N}$ interference signatures. In principle, the poorly known response functions of the detector are already folded into these experimental data sets. Following the conclusions of Sect. 2.2, the basis functions derived from experimental data should be relatively insensitive to the type of media that the photons traversed through. Thus by performing controlled experiments with and without fissile signatures, or with particular interference sources (e.g. teflon to produce $\left.{ }^{16} \mathrm{~N}\right)$, basis functions for each individual source can be constructed.

Due to the poor resolution of the detectors, Descalle's energy response results are used to calibrate the detectors to peaks of known sources, such as the gamma peaks of Yttrium. Roughly speaking, these peaks occur at an energy between the Compton edge and full photo-absorption peak. Details of this calibration are given in Sect. 4. These calibrations are a necessary step in constructing consistent basis functions that reside in the energy-time domain.

Each experimental data set representing a particular source is smoothed via a Savitzky-Golay filter[7, 8] when constructing its basis function. This is necessary as the data sets themselves can be noisy due to Poisson statistics. The parameters of this particular filter are shown in Tab. 2.2. This particular filter has the benefit of smoothing the data while preserving higher moments of peaks in the data set. This is especially important when constructing the ${ }^{16} \mathrm{~N}$ basis function interference peak. In this particular case, preserving the width and height of this peak while smoothing the data is essential in producing a correct half-life of this spectrum in the basis function ${ }^{2}$.

\subsubsection{Background}

Basis functions representing active background (i.e. irradiated cargo without fissile material or interference signatures) as calculated from different experi-

\footnotetext{
${ }^{2}$ This assumes that the data set itself has already been corrected for dead time.
} 
mental runs have very little (if any) structure above $2.5 \mathrm{MeV}$. As Figs. 2.15 and 2.16 show, in this region the spectrum is basically flat regardless of the cargo medium and for all times. As one goes well below $2.5 \mathrm{MeV}$, the picture is quite different as the in-medium activations are quite different depending on the type of cargo.

Because the background spectrum is essentially flat as a function of time in the high energy regime, it is more convenient (and faster) to fit the experimental background to a constant value by using the data points residing at long times ( $t>85$ seconds), as opposed to fitting a background basis function in the entire energy-time domain of the experiment data. This is possible since at such long times, it is expected that fissile signatures and interference signatures will have decayed below background levels. Further details of this fitting procedure is given in Sect. 4. Though this is the preferred method for extracting the background, we show sample basis functions of the background (Figs. 2.15 and 2.16) for completeness.

\subsubsection{HEU}

Figure 2.17 shows an experimental run consisting of a small sample of HEU in three feet of wood, and the corresponding basis function constructed from this data set. Again note the distinctive feature of the HEU signal compared to purely active background runs shown in Figs. 2.15 and 2.16, particularly in the energy range above $2.5 \mathrm{MeV}$. Also note that the signal becomes basically non-existent above $5 \mathrm{MeV}$ for all times.

\subsection{3 $\quad{ }^{16} \mathrm{~N}$ interference}

A teflon source was used to construct the ${ }^{16} \mathrm{~N}$ interference signature through the reaction ${ }^{19} \mathrm{~F}(\mathrm{n}, \alpha){ }^{16} \mathrm{~N}$. The nitrogen would subsequently decay with half-life $\tau=7.13$ seconds predominantly to the first excited state of ${ }^{16} \mathrm{O}$ residing at 6.1 $\mathrm{MeV}$, which would immediately decay to its ground state. The teflon source was immersed directly over the aperture. An example experiment is shown in Fig. 2.18 (a) and the corresponding basis function constructed from this data in Fig. 2.18 (b).

\subsubsection{Other interferences}

In principle there will be other sources of interference signals that occur in the high-energy region above approximately $2.0 \mathrm{MeV}$. Examples of such sources are the $3.084 \mathrm{MeV}$ line of ${ }^{49} \mathrm{Ca}$ and $2.113 \mathrm{MeV}$ line of ${ }^{56} \mathrm{Mn}$. The former can be activated from cargo containing wood, for example, and has a half life of approximately 8 minutes. The latter is present in steel alloys; its half-life is over 2 hours. Church has performed preliminary interference tests and concluded thus far that interference sources other than ${ }^{16} \mathrm{~N}$ will contribute at most at the level of $10^{-5}$ of the active background level. For all results presented here we have only included ${ }^{16} \mathrm{~N}$ as a possible interference. Prussin has argued that the 


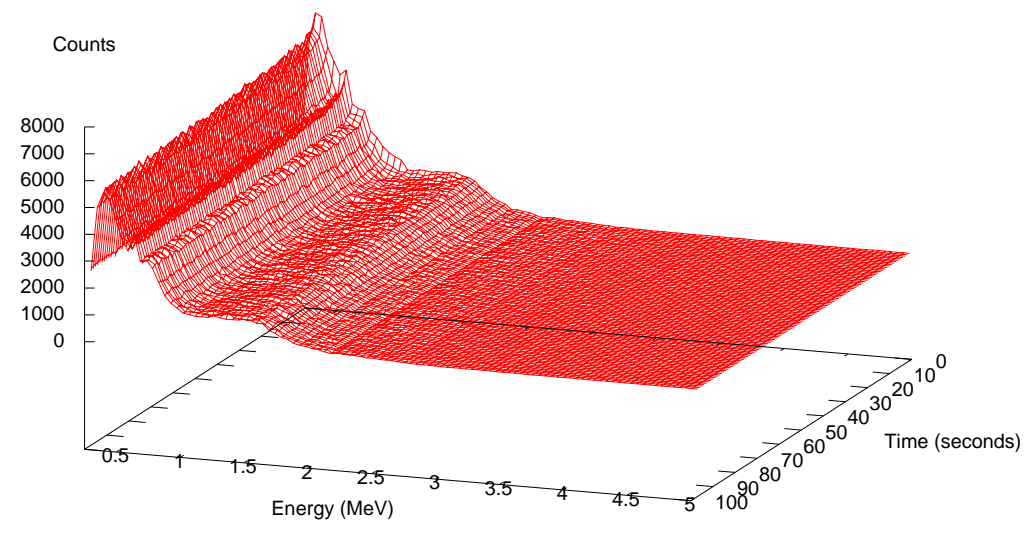

(a)

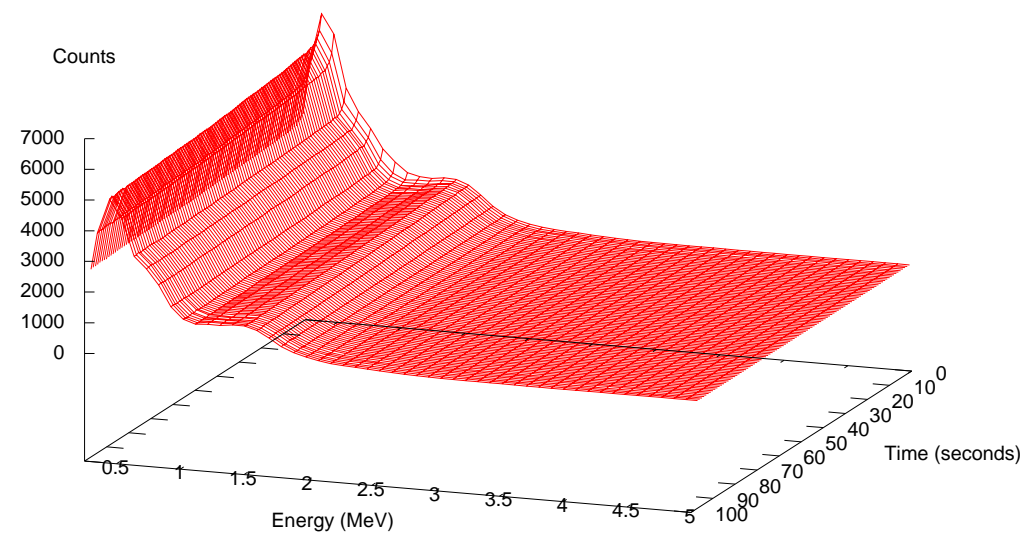

(b)

Figure 2.15: Panel (a) shows an example data set taken after a 30 second irradiation of wood cargo. Panel (b) shows the corresponding basis function constructed from this data set. Note the lack of structure at high energies (> $2.5 \mathrm{MeV})$. 


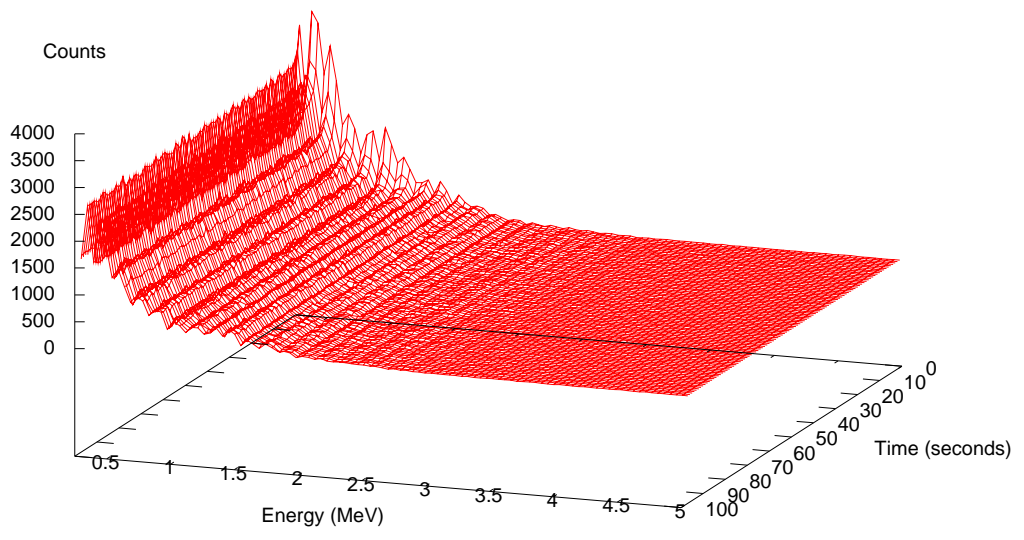

(a)

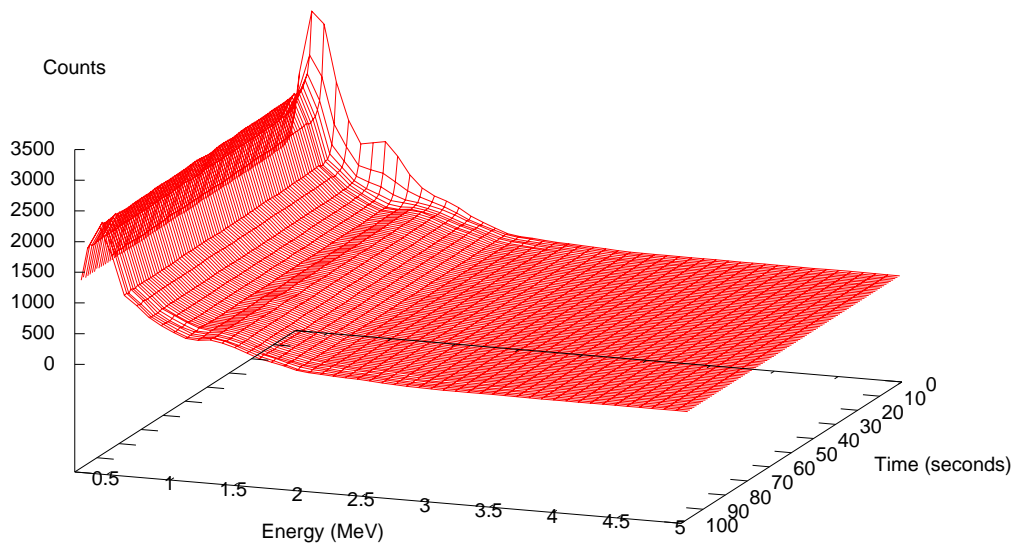

(b)

Figure 2.16: Same as in Fig. 2.15, but with steel cargo. 


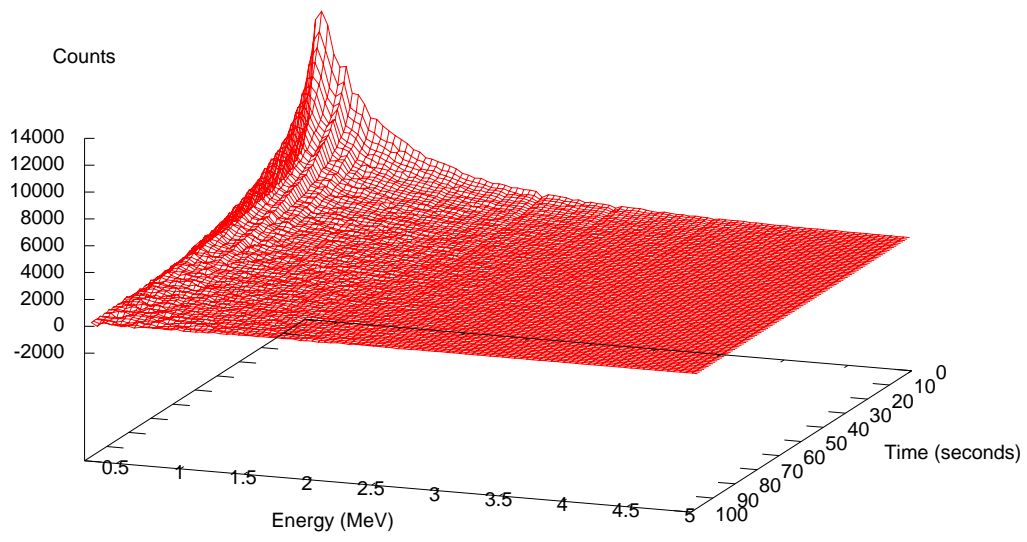

(a)

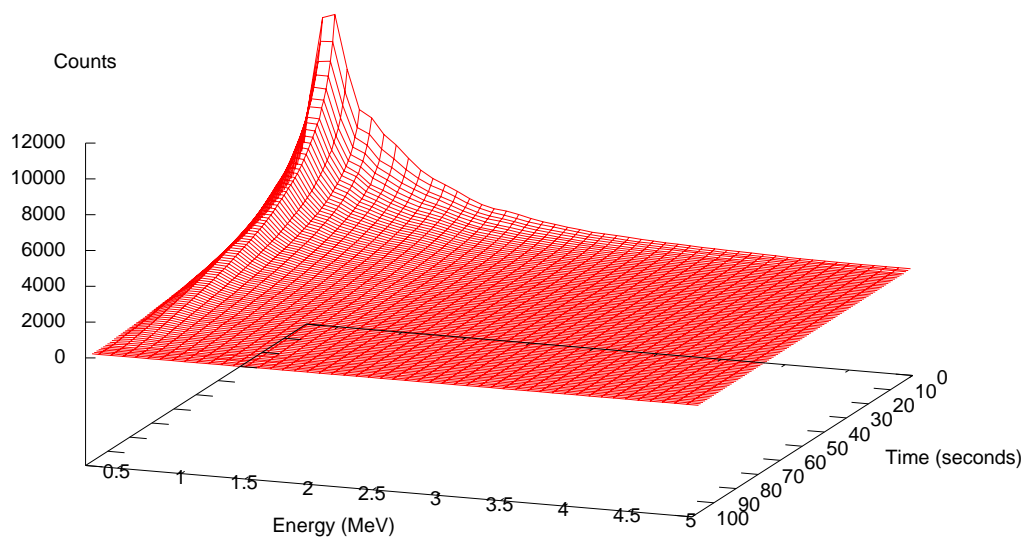

(b)

Figure 2.17: Panel (a) shows an example data set taken after a 30 second irradiation of wood cargo containing a small sample of HEU. Panel (b) shows the corresponding basis function constructed from this data set. 


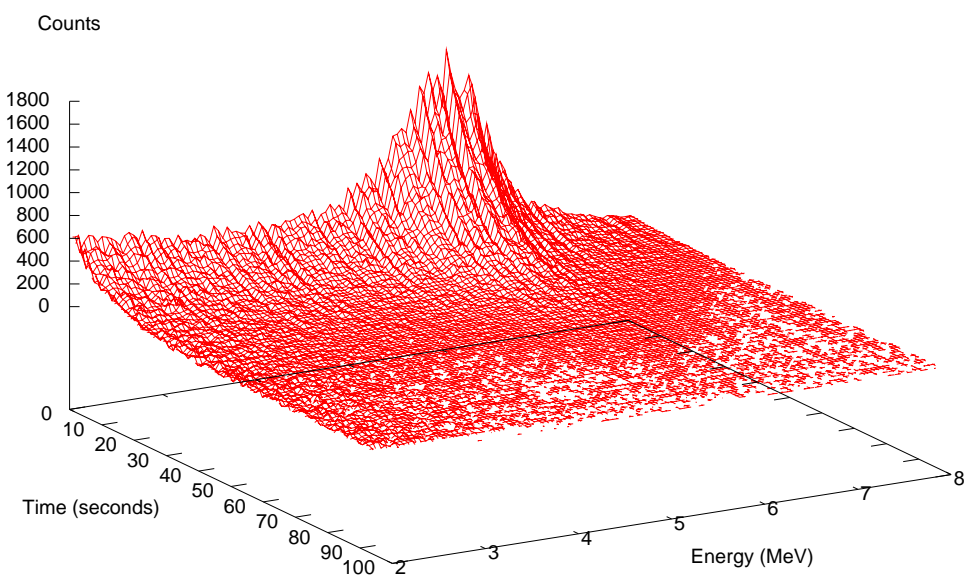

(a)

\section{Counts}

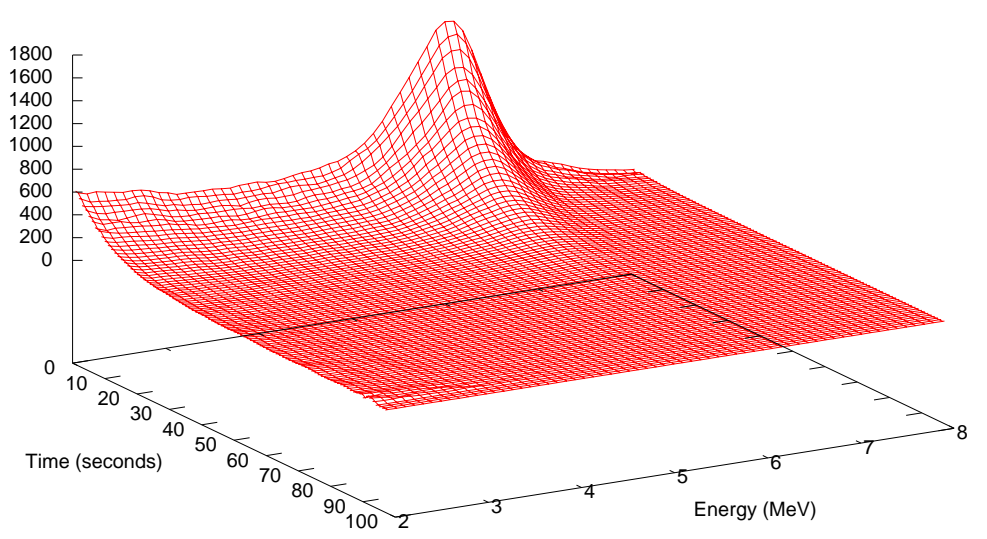

(b)

Figure 2.18: Panel (a) shows a sample experimental signal due to the presence of ${ }^{16} \mathrm{~N}$. Panel (b) shows the corresponding basis function that is constructed from this set. 


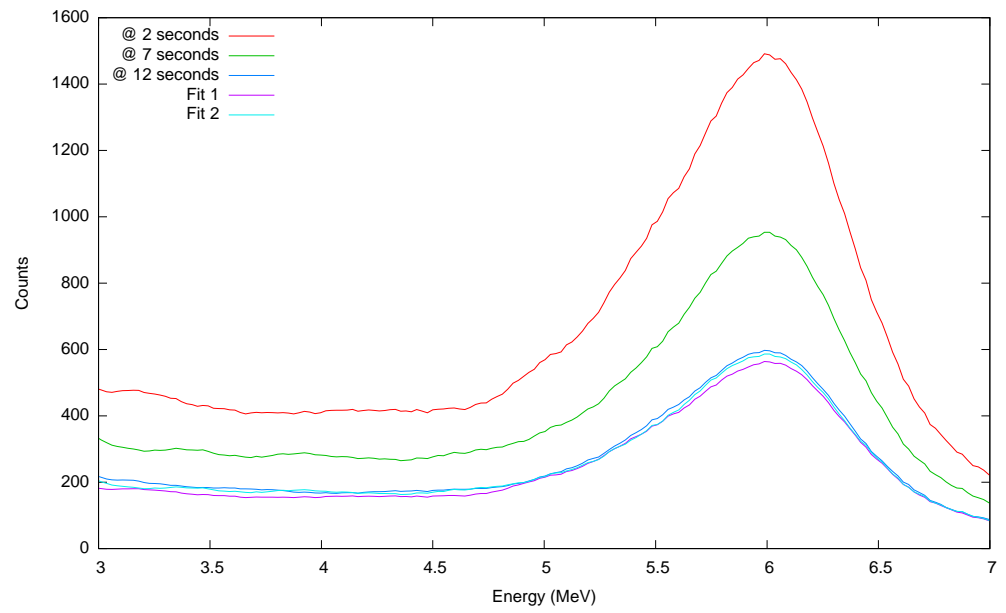

Figure 2.19: Experimental energy-counts profile of ${ }^{16} \mathrm{~N}$ source at 2, 7, and 12 seconds. The data has been smoothed to facilitate presentation. The line labeled Fit 1 uses the profile at 2 seconds to predict the profile at 12 seconds using the known 7.13 second half life of the ${ }^{16} N$ source. Note that it underpredicts the experimental profile. The line labeled Fit 2 uses the profile at 7 seconds to due the same prediction. Here the prediction is much better, indicating that deadtime is less of an issue for this case.

8 minute half-life of ${ }^{49} \mathrm{Ca}$ might could manifest itself in our fits. We plan to investigate this interference in the near future. We do not believe its presence will dramatically alter our current conclusions.

\subsection{Importance of Dead-time Corrections}

Because of the large count rates after irradiation, deadtime issues come into play. As an example, Fig. 2.19 shows the energy-count profile of an irradiated teflon source at times $t=2, t=7$, and $t=12$ seconds using data that has not been corrected for deadtime. The data has been smoothed to de-clutter the figure. Given that the half-life of ${ }^{16} \mathrm{~N}$ is 7.13 seconds, one can predict what the profile will look like at $t=12$ seconds using the raw data at $t=2$ seconds. In doing this one underpredicts the profile, as shown by the line labeled Fit 1 . On the other hand, one can also use the raw data at $t=7$ seconds to predict the same profile. The prediction is closer in this case (Fit 2). These results are indicative of deadtime errors. Church and Manatt have written various routines that can perform deadtime corrections using the ${ }^{*}$.lst files produced from experimental runs. Preliminary analysis shows that deadtime corrections can be large at small times $(<5$ seconds). Table 2.3 shows some typical deadtime errors as a function of time calculated by Church. Unfortunately, due to lack of funding, time constraints, and more pressing matters related to the nuclear car wash, 
Table 2.3: Sample deadtime errors at various times

\begin{tabular}{|c|c|}
\hline \hline Time (seconds) & Deadtime error \\
\hline 8 & $4.81 \%$ \\
16 & $3.49 \%$ \\
28 & $2.92 \%$ \\
\hline \hline
\end{tabular}

most experimental runs have not been corrected for deadtime. This issue will be resolved in the next version of the alarm algorithm.

On the other hand, Norman has suggested utilizing the counts at energies above $8 \mathrm{MeV}$ to estimate deadtime errors. This idea assumes that the counts in this energy regime are due to interactions with high-energy cosmic ray particles and thus should be constant as a function of time and energy. A non-constant count as a function of time would suggest the presence of deadtime errors. By comparing the count at time $t$ with the counts at late times of the data (here the deadtime errors should be zero or constant), one can estimate the relative deadtime error and subsequently the deadtime correction at time $t$. This proposed method is convenient in that the deadtime corrections can be calculated from the data itself.

We investigated the feasibility of this procedure. Figure 2.20 shows the summed counts above $8 \mathrm{MeV}$ as a function of time for a certain experimental run. Because of the low counts and large statistical errors, it is not possible to extract a time-dependence of the counts from these results. Similar results were obtained for other runs. Thus deadtime corrections were not corrected in this manner. If counts are increased in this energy regime (e.g. by using larger detectors), this method should become viable.

To minimize the effects of deadtime errors, we perform our analysis above a lower time cutoff of $t_{c} \sim 5$ seconds. With cutoff times larger than this any fissile signature, if present, becomes much weaker and the signal-to-noise ratio is worsened.

\subsection{Constructing Receiver Operator Character- istic (ROC) Curves}

As mentioned earlier, the intrinsic noise of the experimental data and the uncertainties introduced by the fitting algorithms preclude any definite predictions on the absence or existence of fissile signatures. Interference signals will further cloud the prediction. Thus any prediction will be accompanied with a corresponding uncertainty. For cases where the fissile signature is extremely weak (or where noise is very large), an alarm algorithm may falsely identify the signal as a null result (or falsely predict the presence of fissile materials). Such false negatives (or false positives/alarms) will occur given a large enough sample of experiments. It is therefore necessary to quantify, as much as possible, the probability for these types of occurrences. 


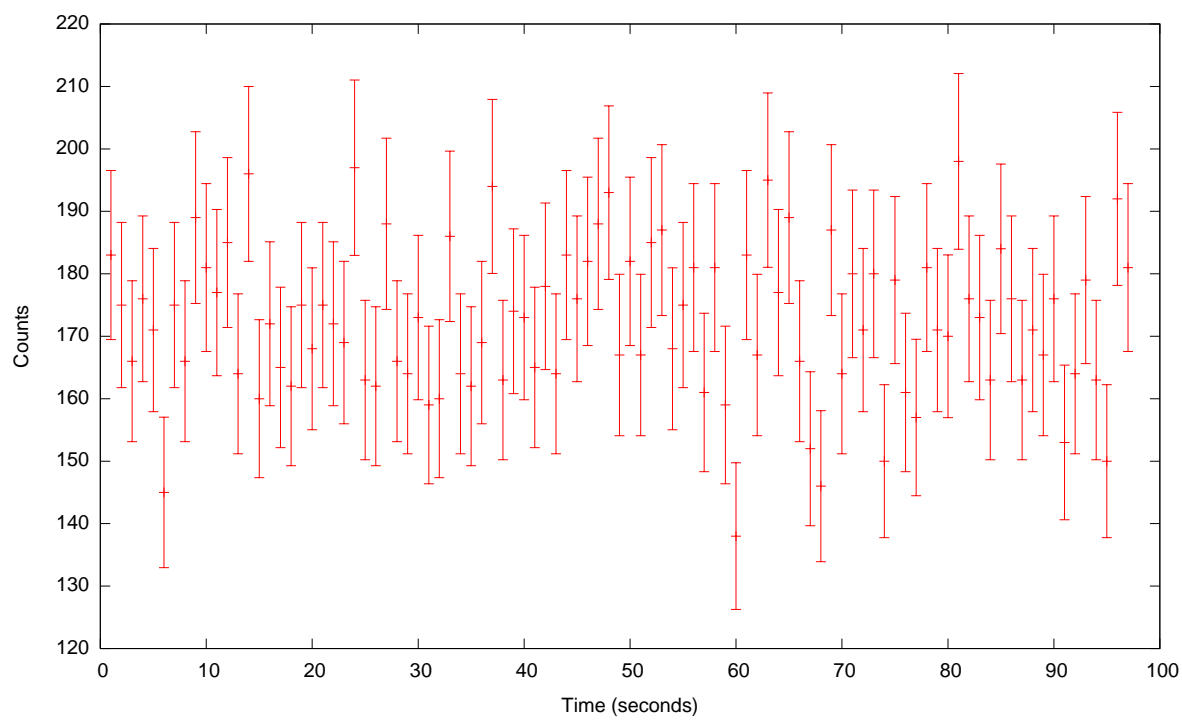

Figure 2.20: Summed counts above $8 \mathrm{MeV}$ as a function of time. The large uncertainties and fluctuations make it difficult to extract deadtime errors using the method proposed by Norman.

Receiver-operator-characteristic (ROC) curves allow for a simplified way to quantify the probability of these occurrences. By measuring the overlap in distribution of null events with the distribution of positive events, false alarm rates (or equivalently, false negative rates) can be calculated without specific knowledge of the underlying statistics intrinsic to the experiment. The construction of ROC curves is relatively straightforward, as long as there is a large sample of null results and positive results from which to construct distributions. The reader is referred to Refs. [9, 10] and references within for further details on the theory behind ROC curves. In Sect. 3.4 we construct the ROC curves from our most sensitive experiment run (HEU in $5 \mathrm{ft}$ of steel) and use them to obtain optimal time windows for performing our analysis as well as setting a delimiter between a null result and a positive result.

\subsubsection{Why bootstrap the data?}

Standard fitting routines, such as least-squares fitting via normal equations or singular-value-decomposition (SVD)[7], have as output the standard deviation $\sigma$ of the fitted parameters, as well as the $\chi^{2}$ of the fit. These parameters assume that the error in the experimental data follows a Gaussian distribution. For our experiments this is not the case, as counting statistics have errors dictated by Poisson statistics. Thus the extraction of uncertainties from run-of-the-mill fitting routines must be done with care.

On the other hand, if there exists large samples of experimental runs, the 
set of fitted coefficients extracted from these samples will form a distribution from which an uncertainty can be extracted. This scenario is more suited for evaluating false alarm rates as well, since ROC curves can be calculated from the overlap of the null distribution with other distributions. Furthermore, the extracted uncertainties will respect the underlying statistics intrinsic to the experiment, since the distributions are calculated directly from the sample of experiments without any prejudices (as opposed to assumed Gaussian distributions for least-squares fitting). This is clearly the desired method in obtaining uncertainties.

Unfortunately, we do not have large enough samples of experiments in which we can form distributions for the fitted coefficients. In this case, given a particular data set, one can statistically sample the data to make pseudo-sets of data. For example, given a data set consisting of $N$ elements, one can make a pseudo-data set of $N$ elements by randomly pulling from the original set $N$ times. One does the sampling with replacement. This ensures that the pseudodata set is not completely identical to the original set, as some of the elements in the pseudo-set will be repeated (on average, approximately $\% 37$ of the data in the pseudo set will be repeated). To make 10 pseudo-sets, for example, one just repeats this scheme 10 times. For each pseudo-set of data, one passes the data through some fitting algorithm to extract a fitted coefficient. With sufficient numbers of pseudo-sets a distribution in the fitted coefficients can be formed. This procedure of constructing distributions of parameters by empirically sampling the original data set is called bootstrapping [7, 11]. The uncertainties extracted from the distributions constructed in this manner should respect the underlying statistics driving the experiment. The ROC curves shown in later sections were calculated from distributions constructed in this manner.

Another benefit of employing the bootstrap method for generating distributions is that in addition to just sampling the experimental data, one can also estimate the contribution to the uncertainty due to fluctuations in external environmental variables, such as temperature. For example, if the calibration of the detectors is known to depend on the temperature, than the contribution to the uncertainty due to this dependence can be estimated by varying the temperature (and thus the calibration) during the bootstrap procedure. We perform these analysis in later sections. 


\section{Chapter 3}

\section{Data Analysis}

We now show examples of our fitting procedure and the distributions of the fit coefficients constructed from our empirical sampling procedure. The basis function representing the HEU signature used in these fits were constructed from data set 20060718_run013, which represents the small HEU sample in 2' of wood. The ${ }^{16} N$ basis function was constructed from data set 20060718_run005. Thus these data sets represent our fiducial runs from which we can compare with subsequent runs. Most fits were done in a time window from 5 to 95 seconds after neutron irradiation and energy window from $2.5 \mathrm{MeV}$ to $5.0 \mathrm{MeV}$.

\subsection{Cargoes with HEU}

Figure 3.1 shows an example fit performed on a experiment run in which the large HEU sample was imbedded in 4' of wood. The fit coefficient obtained in this example is $\alpha_{S N M}=.073422$ with $\chi^{2} /$ d.o.f. $=1.27$. To estimate the uncertainty, we empirically sample the data set as outlined in Sect. 2.7.1. Figure 3.2 shows the distributions obtained in this manner.

In Fig. 3.3 we show distributions obtained from various experimental runs where HEU is imbedded in wood and steel cargoes. This calculations were performed with varying backgrounds. In general, distributions calculated from steel cargoes are wider since the signal-to-noise ratio is poorer than their wood counterparts. This is partly due to the fact that steel does a poorer job of thermalizing the neutrons when compared to wood. Steel also attenuates photons more readily.

\subsection{Samples with Teflon Contamination}

Recent runs were performed where teflon was inserted with the HEU sample to simulate the presences of the ${ }^{16} \mathrm{~N}$ contaminant. In run 20060804_run011, teflon was inserted with HEU in 3 feet of wood. Figure 3.4 shows the data and the subsequent fit of this run. The data is divided into two regions colored green 


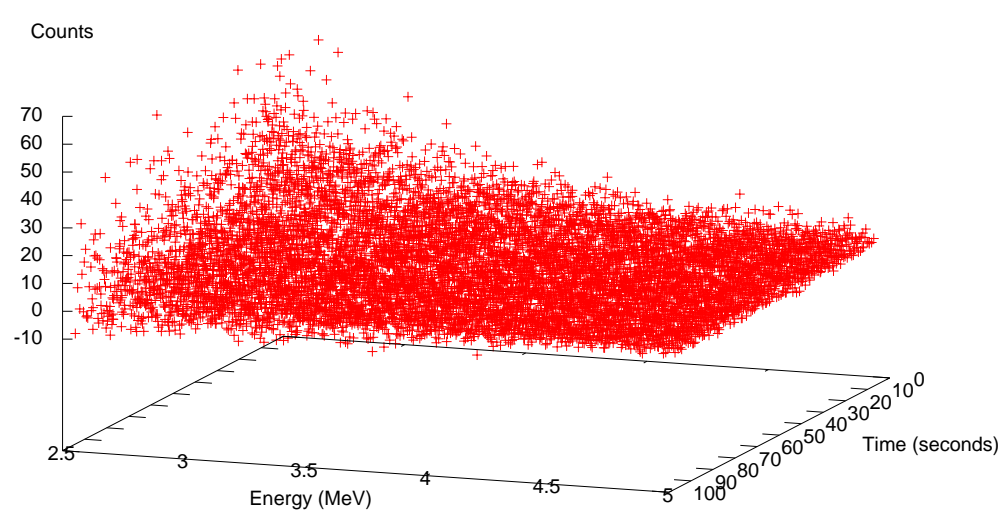

(a)

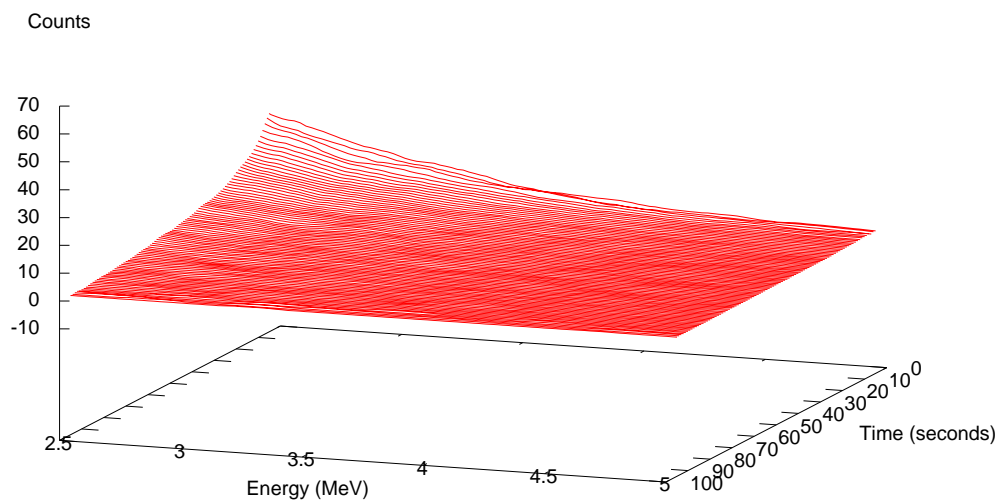

(b)

Figure 3.1: Experimental data from irradiated large sample of HEU in 4' wood (a) and the fitted basis function (b). The fit was performed in energy window [2.5:5.0] MeV, using time window from [5:98] seconds. The fit coefficient for the HEU basis function is 0.073422 with $\chi^{2} /$ d.o.f. $=1.27$. Data set:[20060719_002] 


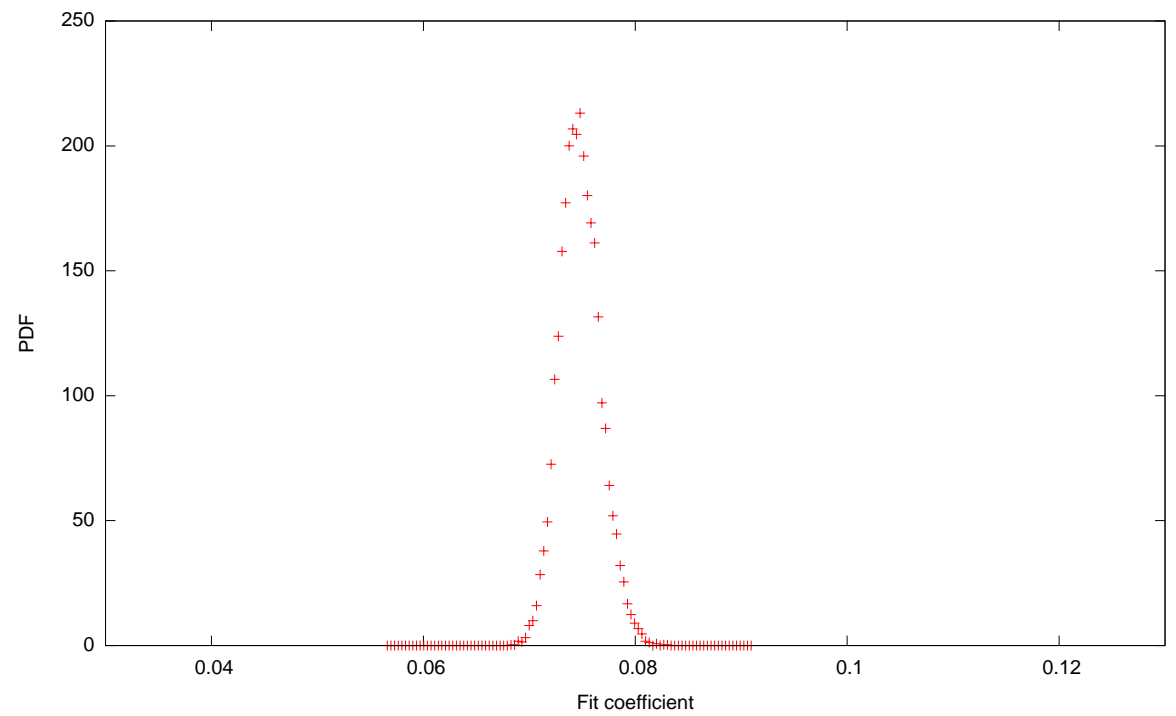

Figure 3.2: Distribution of fitted coefficients using sampling procedure discussed in text.

and red. The green region represents the energy range in which the ${ }^{16} \mathrm{~N}$ is basis function is fitted to, while the red region shows the energy range for performing the HEU basis function fit.

The previous example showed a scenario where the HEU signal was much larger than the ${ }^{16} \mathrm{~N}$ signal. In Fig. 3.5 the situation is reversed. In this experimental run (20061017_run016) HEU is placed in steel. Notice that the fit algorithm failed to detect the presence of HEU in this case. This shows a possible weakness of the alarm algorithm and emphasizes the fact that the presence of large interferences can drastically reduce our sensitivity to detecting fissile signatures. This issue is still not resolved and is currently being investigated.

\subsection{Cargoes without HEU}

One can also apply the sampling procedure to an active background run to obtain a distribution of null results. Figure 3.6 shows such an example for a particular data set. Similar results were obtained for other active background runs. 


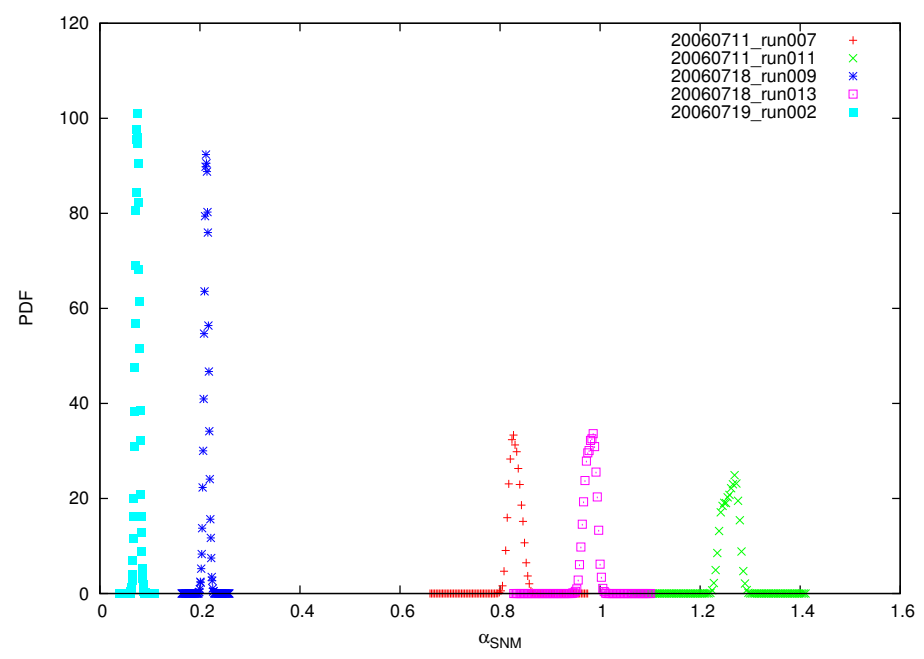

(a)

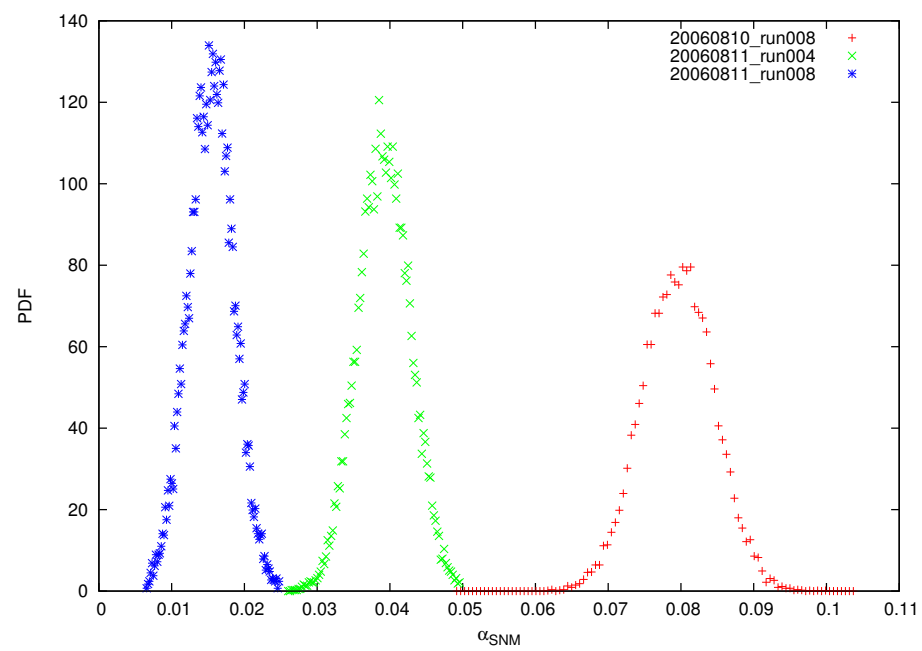

(b)

Figure 3.3: Distribution of fitted $\alpha_{S N M}$ coefficients for various HEU scenarios in wood (a) and steel (b). Note the different scales in the ordinates of the two panels. Data sets are labeled in the figures. 


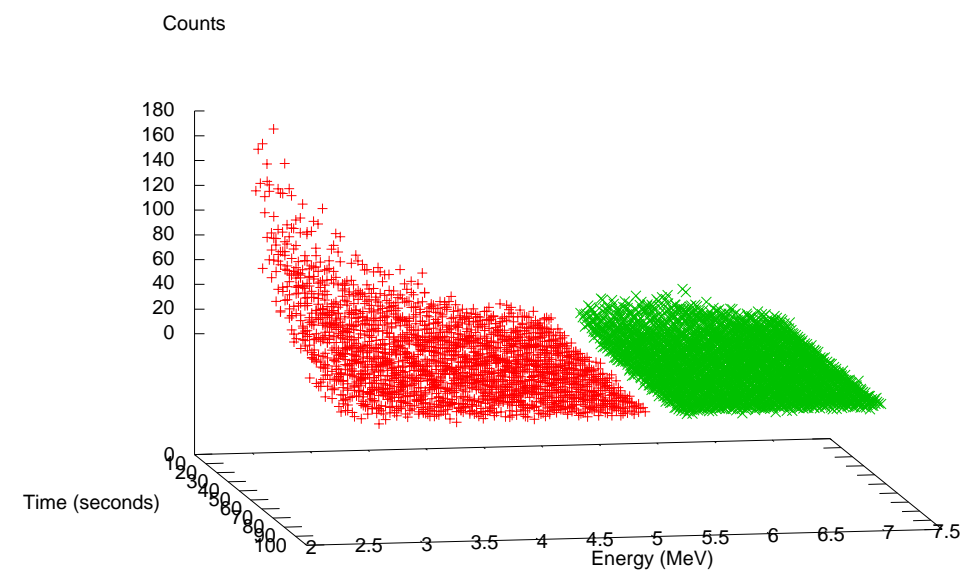

(a)

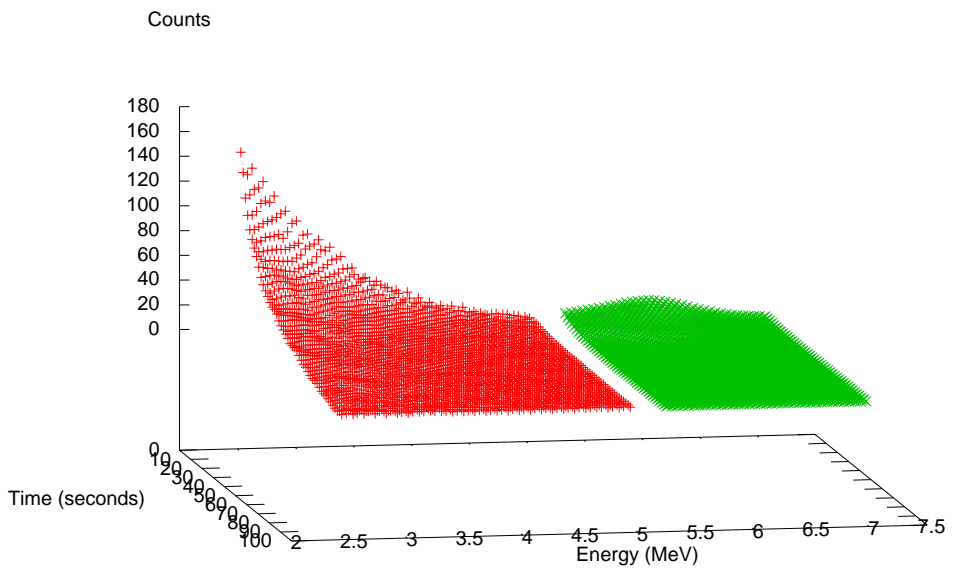

(b)

Figure 3.4: Raw data of run where HEU and teflon are both imbedded in wood to simulate presence of ${ }^{16} N$ contaminant (a). The green points show the region in which the ${ }^{16} \mathrm{~N}$ peak is searched for, while the red points show the region in which the HEU signature is searched. Panel (b) shows the subsequent fitting of the basis functions. Data set:[20060804_run011] 


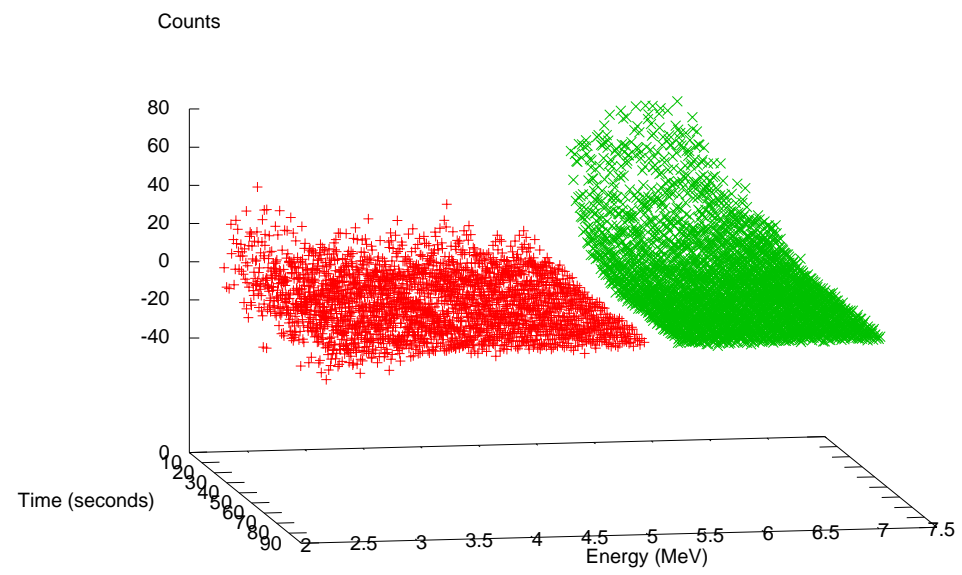

(a)

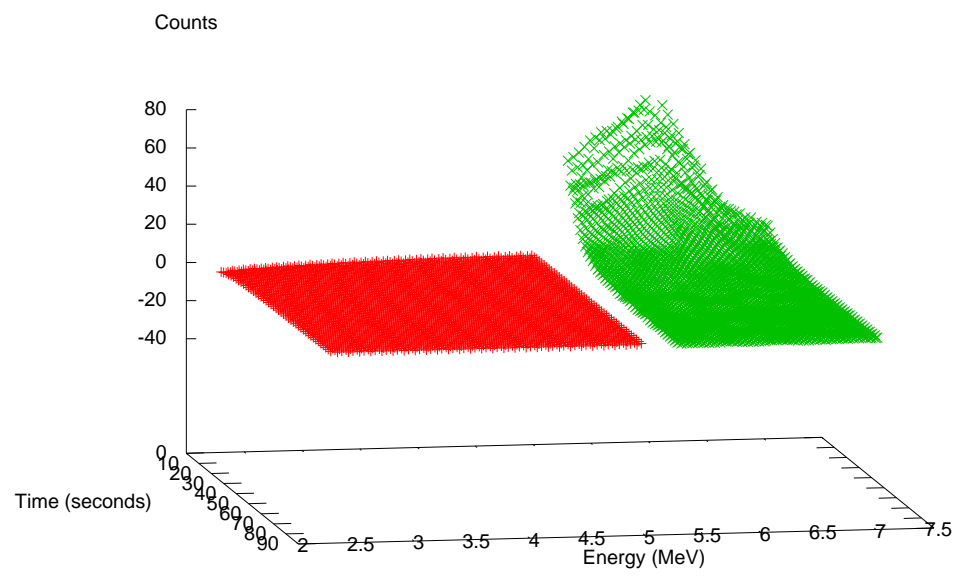

(b)

Figure 3.5: Raw data of run where HEU and teflon are both imbedded in steel to simulate presence of ${ }^{16} \mathrm{~N}$ contaminant (a). The green points show the region in which the ${ }^{16} N$ peak is searched for, while the red points show the region in which the HEU signature is searched. Panel (b) shows the subsequent fitting of the basis functions. Data set:[20061017_run016] 


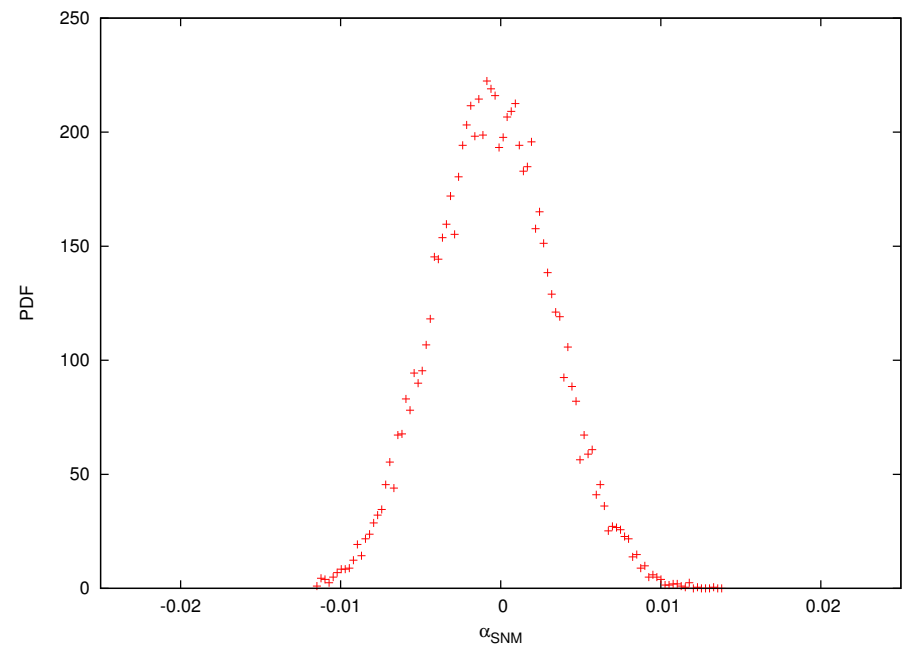

Figure 3.6: Distribution of null events (no HEU) for fitting coefficient $\alpha_{S N M}$. The $\mathrm{y}$-axis has been blown up by a factor of 2 to facilitate viewing. Data set:[20060711_run014] 


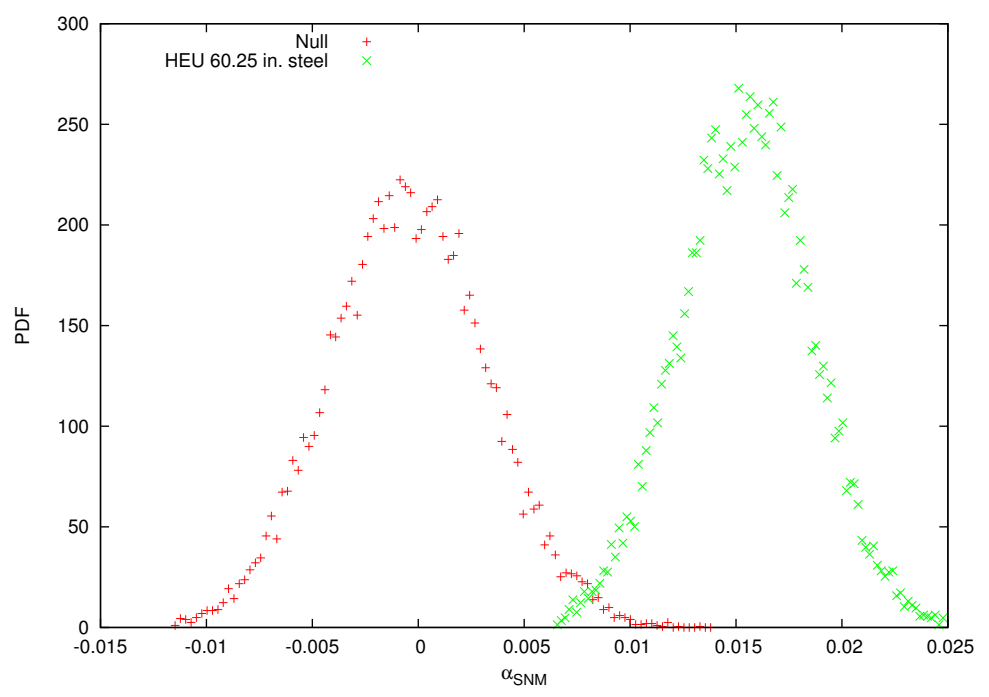

Figure 3.7: Plot shows the overlap of null results and runs generated from data set 20060811_run008, which represents large HEU sample in 60.25" steel. The $\mathrm{y}$-axis has been mulitplied by a factor of 2 to facilitate viewing.

\subsection{ROC curves, false alarm rates, and optimal fitting windows}

To obtain ROC curves, the overlap of the null distribution shown in Fig. 3.6 with distributions shown in Fig. 3.3 must be calculated. However, the only distribution with any appreciable overlap comes from data set 20060811_run008, which represents the large HEU sample in 60.25 inches of steel. The distributions are shown in Fig. 3.7. The corresponding ROC curve is shown in Fig. 3.8. Note that the performance criterion in this case is satisfied as the ROC curve basically intersects the point $(\mathrm{FPF}, \mathrm{TPF})=(.001, .95)$.

We note that run 20060811_run008, in which the large sample of HEU is placed in approximately 5 feet of steel, is our most sensitive run (i.e. has weakest fissile signal). Even so, preliminary analysis suggests that the performance criterion for detecting this weak signal just meets our goals of $95 \%$ success rate with . $1 \%$ false alarm rate. Furthermore, this detection was done on a sample of HEU that has a mass that is almost an order of magnitude smaller than our target of $5 \mathrm{~kg}$. We note that all our wood runs, and runs where there was less steel and the ${ }^{16} \mathrm{~N}$ interference signal is relatively small, easily satisfies our detection criterions. For the remaining sections most of the calculations shown are performed on the run corresponding to the large sample of HEU in 60.25" 


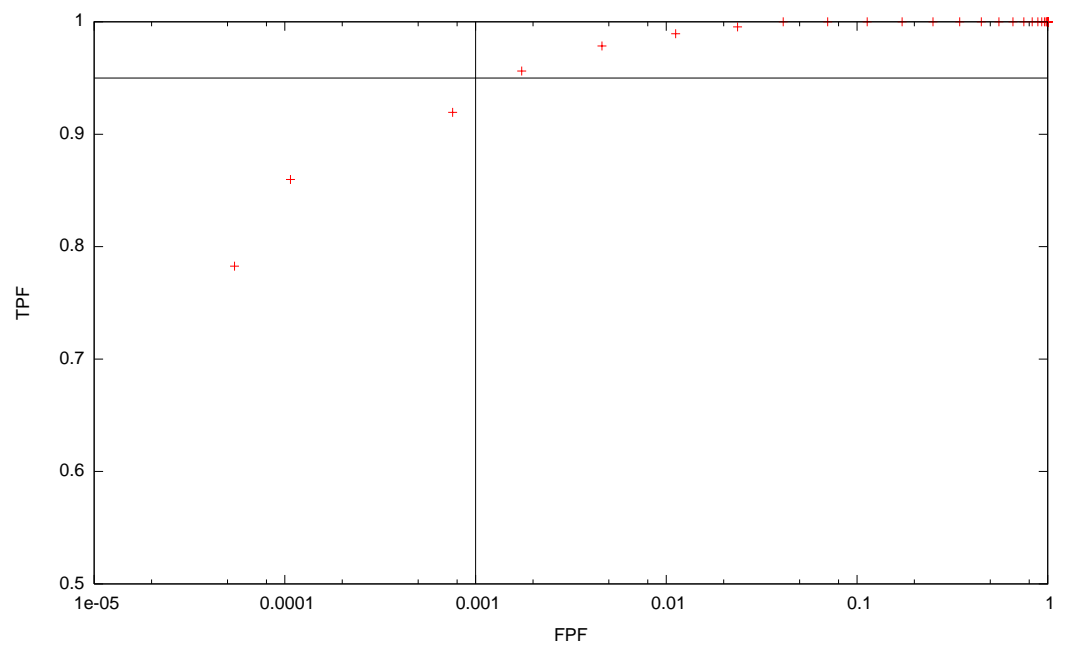

Figure 3.8: ROC curve calculated from overlap of null results with data set 20060811_run008 shown in Fig. 3.7. The black lines corresponding to the intersection of our detection criterions of $95 \%$ success rate and $.1 \%$ false alarm rate. The delimiter that corresponds to this point is $\alpha_{S N M}=.0105$.

of steel.

\subsubsection{Optimal time window}

Is it necessary to use the full 95 seconds to perform our fits? Ideally one would prefer a time window as small as possible. We have performed multiple fits with varying time windows, the ROC curves of which are also shown in Fig. 3.9. For time windows above 85 seconds, the ROC curves are very similar. This suggests that the fissile signal has decayed below background levels at these times. For time windows less than 85 seconds, the ROC curves deteriorate quickly.

One can also look at the integrated signal-to-background ratio as a function of time window, as is shown in Fig. 3.10. Above 85 seconds there is relatively small variation in the signal-to-background ratio, but as one reduces the time window below 85 seconds there is a drastic decrease in the signal-to-noise ratio. These analyses suggest that the optimal time window is approximately 85 seconds. We stress that our analyses have been done on cargo that has been irradiated for 30 seconds. It is likely that the optimal time window will be a function of irradiation time ${ }^{1}$. We have not done repeated our analyses on cargo irradiated for shorter times. We plan to do so in the near future.

\footnotetext{
${ }^{1}$ It is likely that it will also depend on the strength of interference signals as well.
} 


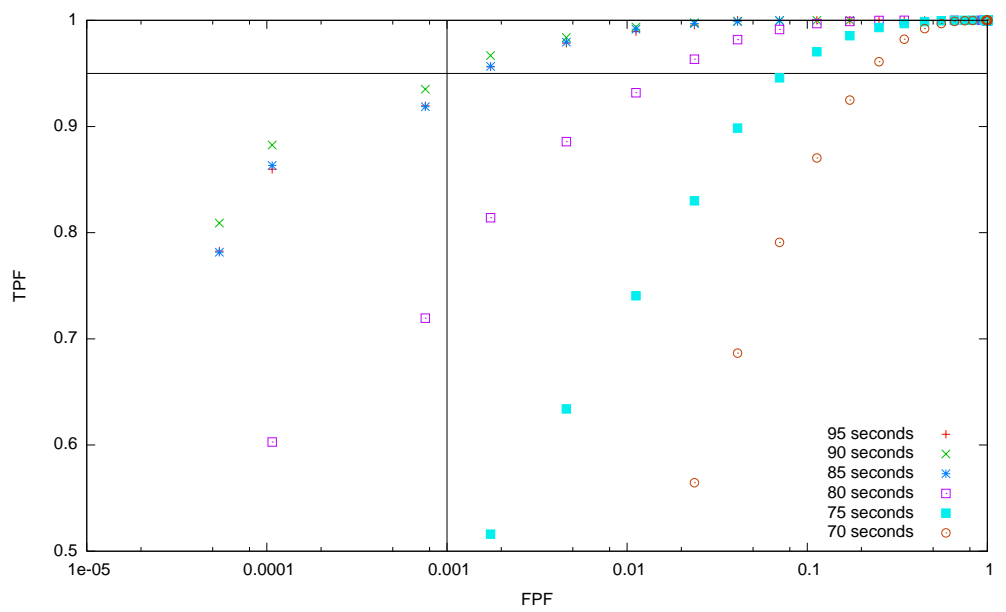

Figure 3.9: ROC curves calculated from overlap of null results with data set 20060811_run008 using different time windows for performing fits, as labeled in figure. For time windows of 85, 90, and 95 seconds, the performance criterions are met (.95 TPF and .001 FPF). For time windows below 85, the ROC curves deteriorates quickly due to loss of signal.

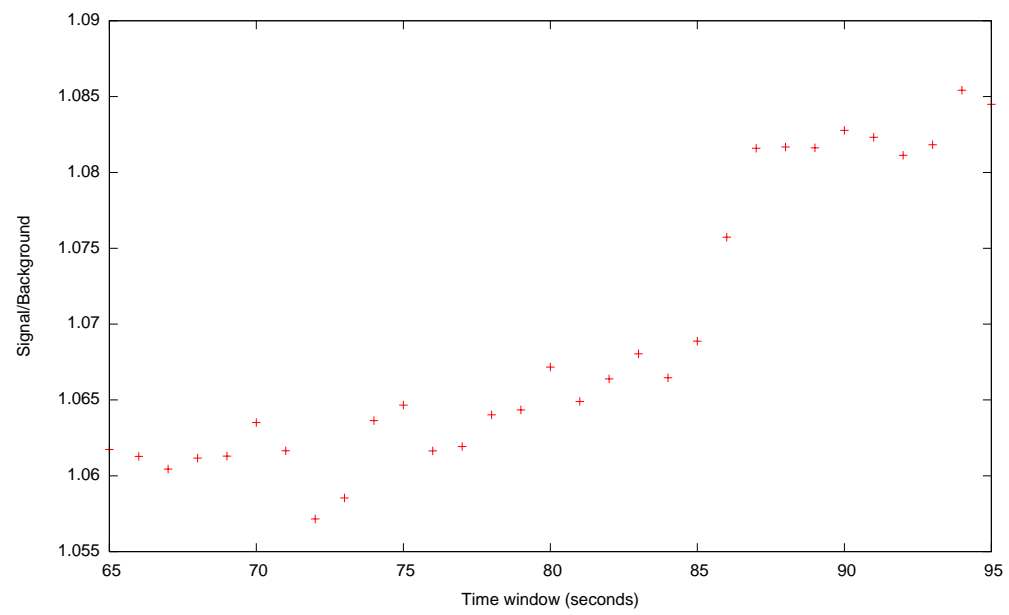

Figure 3.10: Signal-to-background ratio as a function of time window. Data set:[20060811_run008] 


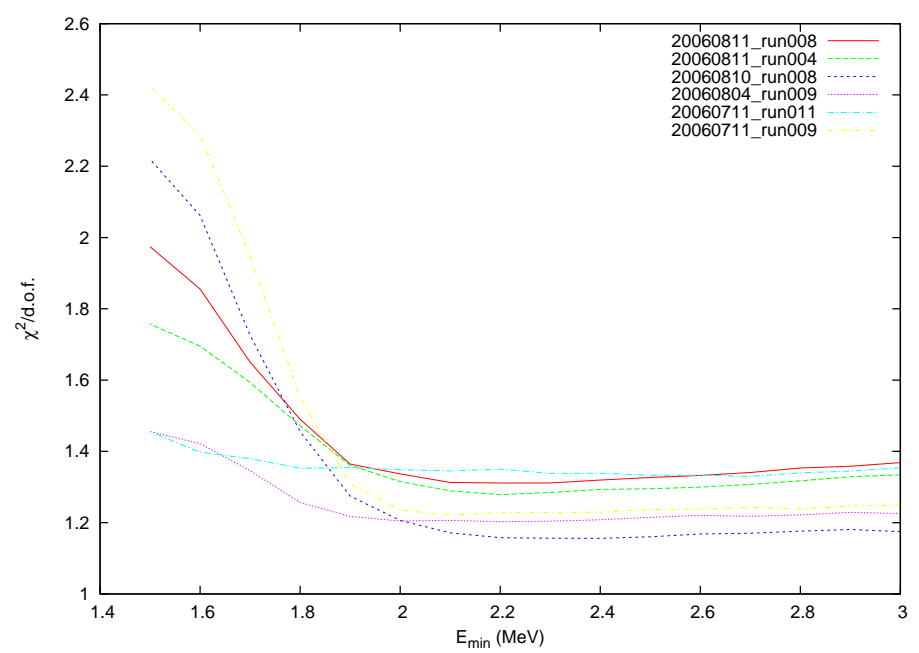

Figure 3.11: $\chi^{2} /$ d.o.f. of fits as a function of lower energy cutoff. Upper energy cutoff is fixed at $5.0 \mathrm{MeV}$. Data sets are labeled in the plot and represent various HEU runs imbedded in wood and steel.

\subsubsection{Optimal energy window}

Determining an optimal energy window is much more difficult. This is partly due to the fact that the amount of in-medium activation strongly depends on the type of cargo. As one goes below $3 \mathrm{MeV}$ in the spectrum, there are various shortlived products that can be activated via neutron interactions. Such activations in the 2.5-3.0 MeV range did not seem to adversely affect our fits thus far. However, we have only looked at two types of cargo: Wood and steel.

An initial guess at determining an optical energy window is to look at the performance of our fits as a function of energy window. Figure 3.11 shows the $\chi^{2} /$ d.o.f. as a function of lower energy cutoff. All calculations were done with a fixed upper energy of $5.0 \mathrm{MeV}$. As one lowers the energy cutoff below $3 \mathrm{MeV}$, the performance of the fit improves initially. This is partly due to the fact that the signal becomes stronger. However, as one goes below 2.2$2.3 \mathrm{MeV}$, the fit starts to drastically worsen for the runs that involved steel cargoes (20060810_run008, 20060811_run004, 20060811_run008). Here the long-lived in-medium activations of the cargo start to interfere with the HEU signature, causing the performance of the fit to quickly worsen. For the wood runs (20060711_run009, 20060711_run011, 20060804_run009), the fits seem to deteriorate below a lower bound of $2.0 \mathrm{MeV}$. Thus it would seem that the optimal lower cutoff on the energy window is constrained by our steel runs and is approximately $2.3 \mathrm{MeV}$. We have looked at the performance of the fit as a 


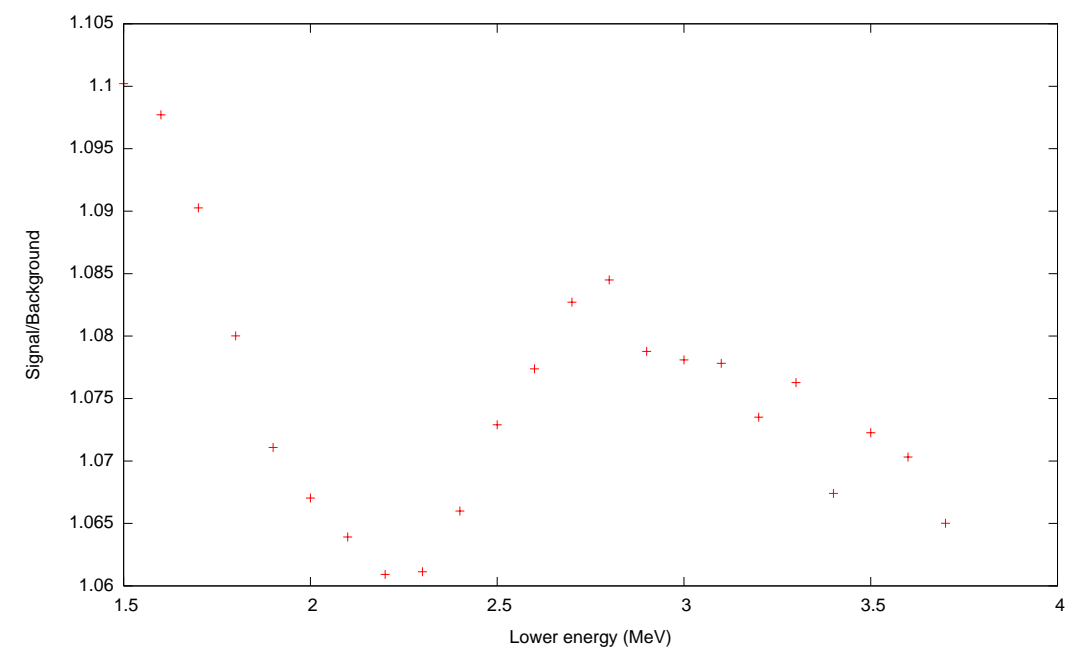

Figure 3.12: Signal-to-background ratio as a function of lower energy cutoff. Data set:[20060811_run008]

function of upper energy cutoff being larger than $5.0 \mathrm{MeV}$. We found little if any dependence. Here the fissile signature is so weak that there is practically no dependence on the upper energy cutoff.

However, if one looks at the integrated signal-to-background ratio, as shown in Fig. 3.12, the ratio seems to peak at approximately $2.8 \mathrm{MeV}$. It worsens in the 2.2-2.3 MeV range, and improves below $2.0 \mathrm{MeV}$ due to the large long-lived in-medium activations. This seems to contradict the results shown in Fig. 3.11, since the optimal lower cutoff in this case seems to be approximately $2.8 \mathrm{MeV}$ as opposed to $2.3 \mathrm{MeV}$. Norman has noted that the $\chi^{2} /$ datum, as shown in Fig. 3.11, is not a good figure of merit for determing the optimal lower energy cutoff since the number of fitted data points is large. Furthermore, Prussin has stressed that even though the short-lived activated products do not seem to be apparent in our fits thus far, it is prudent to keep the cutoff as large as possible so as to minimize their effects. To be safe, we have tentatively stated that the optimal lower-energy cutoff should be approximately $2.8 \mathrm{MeV}$. We note that this value was determined from fits using time windows from 5 to 95 seconds.

The drawback of increasing the lower-energy cutoff is that the spectrum of any present fissile signature is reduced. An alternative option is to delay our analysis to later times (e.g. 15 seconds instead of 5 seconds after end of irradiation) when the short-lived activated products will have decayed below background levels. It may be possible to now lower the energy cutoff well below $2.8 \mathrm{MeV}$. Of course, by working at delayed times the overall fissile signature is reduced. Thus there should be an optimal delayed time and corresponding lower-energy cutoff. Determining these parameters is our next immediate goal. 


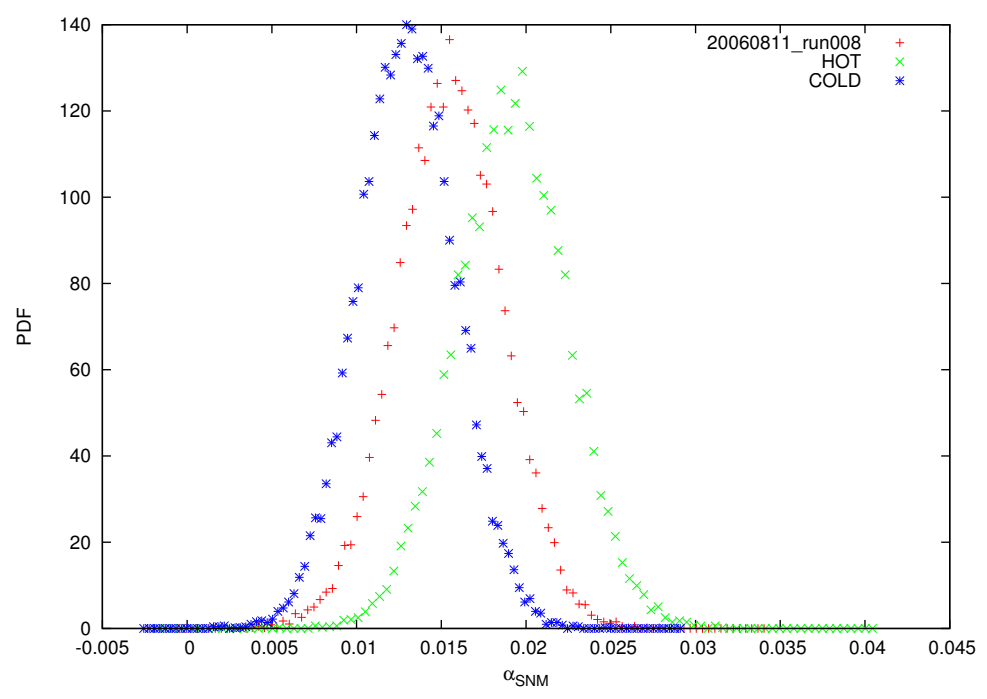

Figure 3.13: Fit distribution of run 20060811_run008 shown in red. HOT and COLD points represent how distribution is altered by adjusting temperature of detectors \pm 3 degrees centigrade assuming $1.2 \%$ change in gain per centigrade.

\subsection{Dependence on temperature fluctuations}

Petersen has made measurements on the dependence of the detector gain (keV /channel) on external temperature and has deduced a $1.2 \%$ change in detector gain per Centigrade relationship. To simulate the effects of this dependence, we have re-calculated our fit distributions for the run 20060811_run008 (large sample of HEU in approximately 5 feet of steel) assuming a \pm 3 centigrade change in detector temperature. Figure 3.13 shows how the distributions change as one increases or decreases the temperatures of the detectors in this fashion. Notice that as one lowers the temperature the signal is artificially reduced. In a corresponding fashion the signal is artificially increased as one increases the temperature of the detectors. Figure 3.14 shows how the corresponding ROC curves are altered with these temperature changes. Our performance criterions are not met when the detectors are cooled in this example. Thus we conclude that for very weak signals the outcome of our fits can be affected by temperature fluctuations.

A possible way to overcome these temperature issues is to constantly immerse the detectors (even during scanning scenarios) to sources that have long halflives and gamma lines below the $2.8 \mathrm{MeV}$ lower energy cutoff. Assuming that the temperatures of the detectors remain constant during the scanning period, 


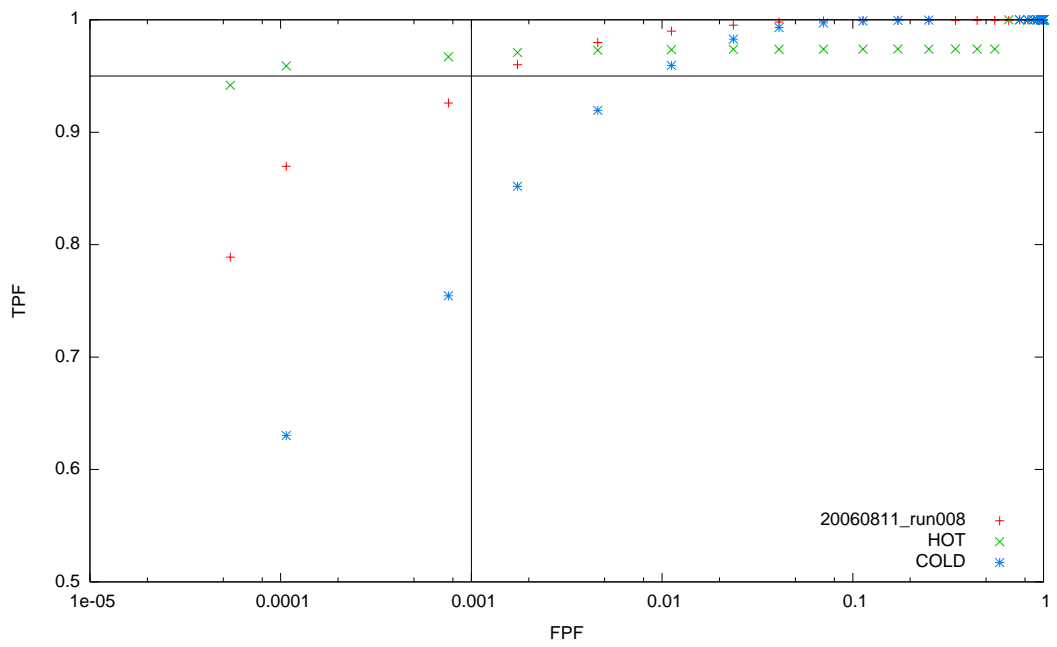

Figure 3.14: ROC curves calculated from distributions shown in Fig. 3.13.

these sources can serve as calibration beacons during data collection. Ideal sources are the two gamma lines of Yttrium. 


\section{Chapter 4}

\section{Coding Logic and Procedures}

The algorithm ALARMA actually consists of three distinct algorithms. The first algorithm, calibrateAlarma, reads in experimental calibration data (Yttrium and teflon) and determines the calibration coefficients that relate data channels to energy. It writes the calibration coefficients out to a file. The second algorithm, basisAlarma, loads in experimental data sets and constructs basis functions from these sets. The last algorithm, fitAlarma, takes a user-chosen experimental data set and fits basis functions to the data. It also performs error analysis by constructing distributions of the fitted parameters using the sampling procedure described in Sect. 2.7. We now describe the individual routines in more detail.

\section{1 calibrateAlarma}

Almost all experiments have accompanying calibration runs where Yttrium and teflon are used as sources with known energy peaks to calibrate the data channels. Yttrium has two gamma lines at $898 \mathrm{keV}$ and $1.8 \mathrm{MeV}$ with long half-lives. Since the Yttrium lines are naturally occurring, no previous neutron irradiance is needed. Teflon sources, on the other hand, require irradiation so that the reaction ${ }^{19} \mathrm{~F}(n, \alpha){ }^{16} \mathrm{~N}$ occurs (1.6 MeV threshold). ${ }^{16} \mathrm{~N}$ subsequently decays with half-life $\tau=7.13$ seconds predominantly to the $6.1 \mathrm{MeV}$ excited state of ${ }^{16} \mathrm{O}$, which decays to its ground state almost immediately. It is these three gamma lines $(.898,1.8,6.1 \mathrm{MeV})$ that calibrateAlarma uses to calibrate the data channels.

Because of the poor detector resolution and the small probability that the photon lines mentioned above are fully deposited in one scattering within the detector, detected peaks due to these lines actually reside lower in energyapproximately half way between the Compton edge and the photon lines. Descalle's simulations show that these lines will be detected as peaks at the energies given 
Table 4.1: Calibration peaks for Yttrium and Nitrogen

\begin{tabular}{|c||c|c|c|}
\hline \hline Element & Yttrium & Yttrium & Nitrogen \\
\hline Line & $898 \mathrm{keV}$ & $1.836 \mathrm{MeV}$ & $6.128 \mathrm{MeV}$ \\
Detected peaks & $799 \mathrm{keV}$ & $1.724 \mathrm{MeV}$ & $6.005 \mathrm{MeV}$ \\
\hline \hline
\end{tabular}

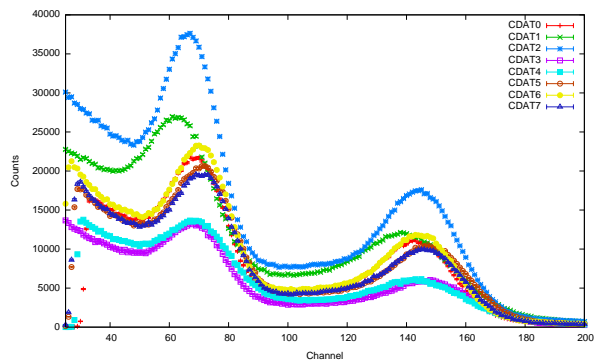

(a)

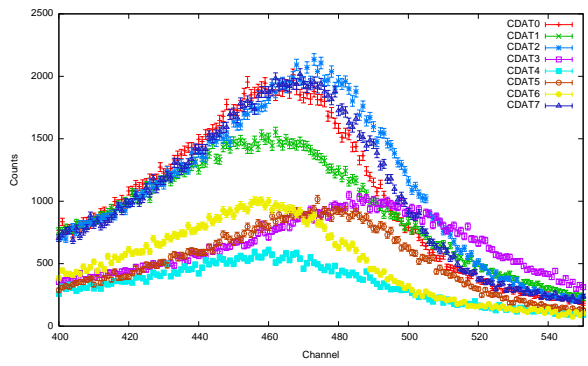

(b)

Figure 4.1: Calibration runs using Yttrium background source (a) and irradiated teflon source $\left({ }^{16} N\right)(\mathrm{b})$.

in Tab. 4.1. calibrateAlarma reads in calibration data with peaks given as a function of data channels. For example, Fig. 4.1 show peaks of eight detectors for runs with an Yttrium background source (panel (a)) and an irradiated teflon source (panel (b)). Note that the peaks do not line up for both calibration runs.

With appropriate input parameters, calibrateAlarma will search for two of the three peaks shown in Fig. 4.1 and map a linear function to the data such that the two peaks reside at their corresponding energies shown in Tab. 4.1. It will do this for each detector. As an example, Fig. 4.2 shows the same data but calibrated using the two Yttrium peaks. The ordinate represents energy $(\mathrm{MeV})$ in this case. Notice that in this case, all peaks in panel (a) line up, but panel (b) still has small variations. The small variations in panel (b) suggest that the calibration is almost linear.

Lastly, calibrateAlarma can perform a quadratic fit using all peaks shown in Fig. 4.1. In this case, all peaks line up at their corresponding energies of Tab. 4.1, as Fig. 4.3 shows. The quadratic coefficients in these fits are a couple orders of magnitude smaller than the linear coefficients and offset coefficients. Again, this points to the fact that detector calibrations are almost linear.

The calibration coefficients are written to a file, which can be subsequently used by basisAlarma and fitAlarma. The following shows a sample input file calibrate.ini that is called by calibrateAlarma:

calibrate.ini

0,1 1 means perform fit, 0 means do not perform fit 


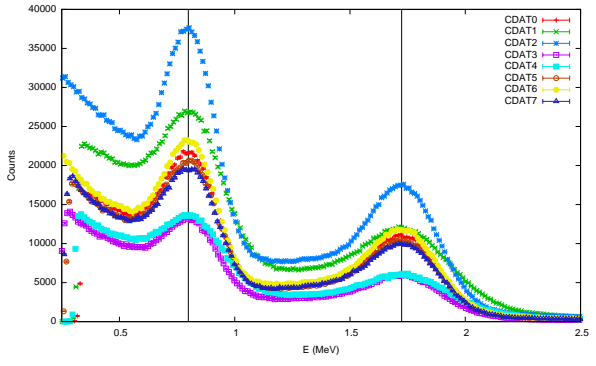

(a)

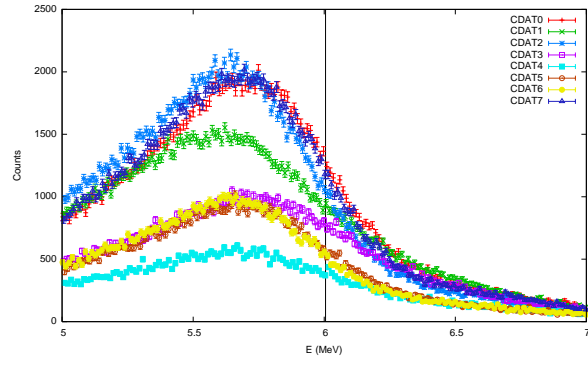

(b)

Figure 4.2: Calibration runs using Yttrium peaks to fit a linear function. Panel (a) shows the Yttrium peaks, which now all line up. Panel (b) shows the irradiated teflon source $\left({ }^{16} N\right)$ (b).

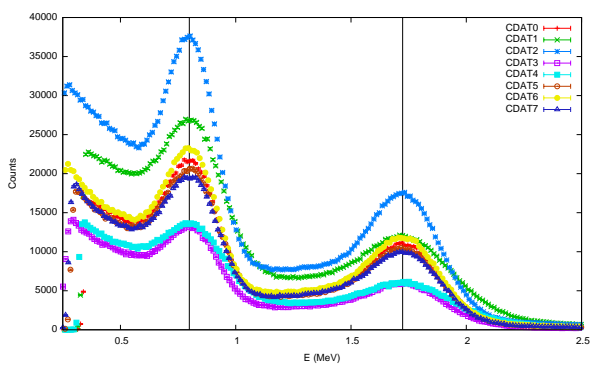

(a)

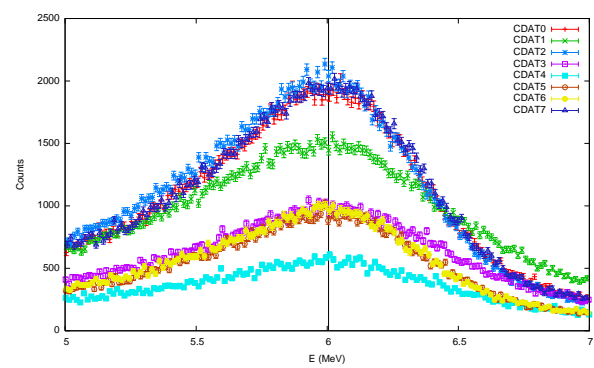

(b)

Figure 4.3: Calibration runs using all three peaks to fit a quadratic function. Panel (a) shows the Yttrium peaks, which now all line up. Panel (b) shows the irradiated teflon source, which all line up as well. (b). 


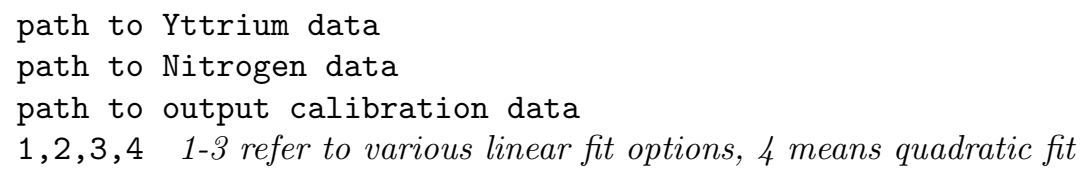

\section{2 basisAlarma}

The routine basisAlarma reads in a user-selected data set and constructs a basis function from the data. In principle, the user should select data with a known source (without contaminants) so that the basis function represents the spectrum from only that source. The basis function is constructed by simply smoothing the data using a Savitzky-Golay filter[8]. When constructing the basis function from the data, an energy interval of $24 \mathrm{keV}$ is used to re-bin the summed data from all available detectors. This binning interval can be easily changed within the algorithm. The entire channel-time domain is used in constructing the basis function even though, as will be described in the next section, only a certain range of this domain will be used in performing the fits. The basis function is written out to a file as specified by the user. Supporting data is also written out in the file, such as cubic spline coefficients used to interpolate the basis functions. Note that the basis functions will be stored in the energy-time-counts domain. It is necessary that the calibration of the detectors (i.e. calibrateAlarma) be performed before using this routine. The following shows the structure of a sample input file basis.ini, which is called by basisAlarma.

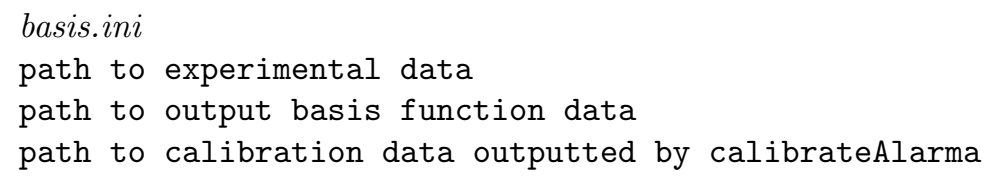

\section{3 fitAlarma}

The crux of the algorithms resides in fitAlarma. After reading in calibration data and basis functions, as well as the experimental data in question, the routine fits the basis functions to the data to obtain fit coefficients. The coefficients measure the amount in which a particular irradiated source is present in the data. The fitting is done in two steps: first a ${ }^{16} \mathrm{~N}$ signal is searched for in the 5.3-7.0 MeV range. This signal, if found, is then subtracted from the data. The fissile signature is then searched within an energy range defined by the user (e.g. 2.5-5.0 MeV) using the subtracted data. The user also has the freedom to choose the time window in which to perform the analysis. This two-stepped procedure of fitting the data was found to give the most robust and consistent fit coefficients. The fitting is done by weighted least-squares (variances are evaluated by square root of the counts), using a singular value decomposition (SVD) 
[7] algorithm. Uncertainties in the fit coefficients are obtained by statistically sampling the experimental data, as described in Sect. 2.7.1.

The following sample input file fit.ini called by fitAlarma shows the various options available to the user. The fit coefficients, as well as their distributions, are written out to file defined by the user. Numerous other write to files are performed to support future graphical displays of ALARMA.

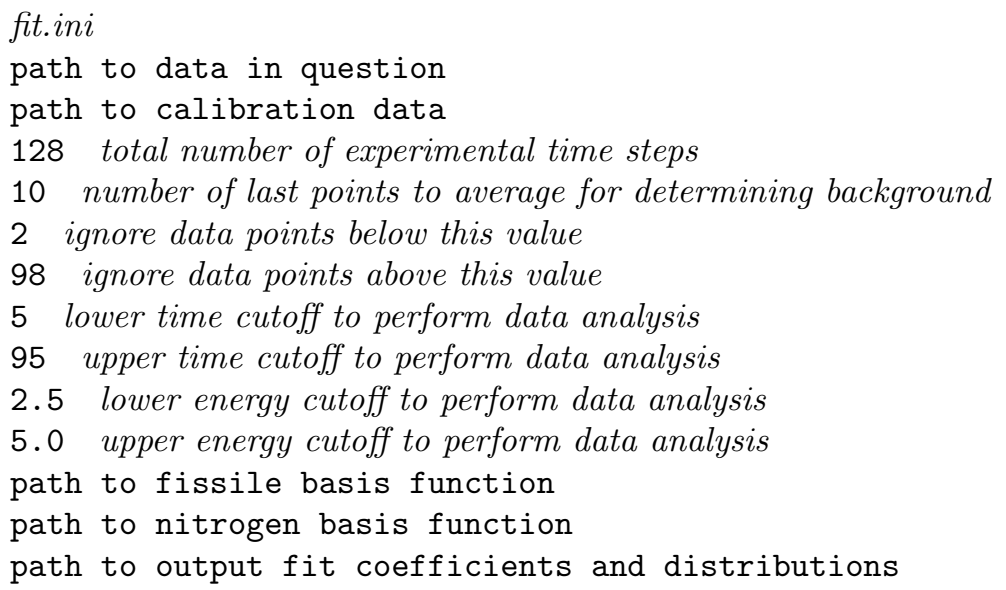




\section{Chapter 5}

\section{Conclusion}

We have developed an alarm algorithm ALARMA for the nuclear car wash experiment at Lawrence Livermore National Laboratory. By utilizing the entire energy-time-counts domain of the experimental data, we were able to easily identify fissile signatures as well as the ${ }^{16} \mathrm{~N}$ interference signature for a wide range of experiments that were performed. For most experimental runs we were able to meet our detection criterions of $95 \%$ success rate and $.1 \%$ false alarm rate.

By looking at our most sensitive runs where the large HEU sample was placed in approximately 5 feet of steel, we were able to deduce the optimal time window of scanning to be no less than 85 seconds (assuming 30 seconds of neutron irradiation). The optimal lower cutoff in energy was more difficult to quantify, as there was a lack of a proper figure of merit in this case. Here the possible presence of short-lived activated products complicated matters. By looking at the integrated signal-to-background ratio, we have conservatively set the lower energy cutoff to be approximately $2.8 \mathrm{MeV}$. This uncertainty in this value should lessen as we analyze more data that has been recently acquired.

We stress that our runs were performed with HEU samples that were smaller by an order of magnitude than our target 5 kilograms. The fact that our detection criterions were met for all our wood cargo runs and most steel cargo runs gives us confidence that detection of 5 kilograms of fissile material during normal field operations should be attainable. This assumes that the nuclear car wash is used as a secondary screening procedure.

\subsection{Things to be done}

An open question is whether the presence of large interfering signals can confuse our detection algorithm. This seems to be the case for the particular run shown in Fig. 3.5. A possible resolution to this problem is to take advantage of the different decay times of the interfering signal (in this case ${ }^{16} \mathrm{~N}$ ) and the fissile signature. By delaying our analysis by a couple of half-lives (7.13 seconds) 
of the interfering source, we can reduce its signature and thereby minimize its interference. This should allow our fitting algorithm to readily detect any underlying fissile signature. This investigation is currently being done.

For extremely weak signals for which our detection criterions are just met, there is concern about the (lack of) robustness of our calculated ROC curves. Already we have shown an example where temperature fluctuations of the detectors by a few degrees can alter our ROC curves. We intend to do more variability tests to quantify, as best as possible, the robustness of our alarming procedure in the case of very weak signals.

Recent data was taken using neutron irradiation times less than 30 seconds. We intend to analyze this data and determine new optimal time and energy windows in which to perform our analyses. 


\section{Bibliography}

[1] D. Slaughter et al., UCRL-TR-214636 (2005)

[2] D. Slaughter et al., UCRL-ID-155315 (2003)

[3] D. Slaughter et al., Nucl. Instr. and Meth. B 241, 777 (2005)

[4] E. .B. Norman et al, Nucl. Instr. and Meth. A 521608 (2004)

[5] J. D. Jackson, Classical Electrodynamics, John Wiley \& Sons (1975)

[6] J. A. Church et al., Nucl. Instr. and Meth. A, CAARI proceedings (2006)

[7] W. H. Press et al, Numerical Recipes in C, Cambridge Univ. Press (1992)

[8] A. Savitzky and M. J. E. Golay, Anal. Chem. 36 1627-1639 (1964)

[9] J. Hanley and B. McNeil, Radiology 143 29-36 (1982)

[10] J. Hanley and B. McNeil, Radiology 148 839-843 (1983)

[11] B. Efron, The Jackknife, the Bootstrap, and Other Resampling Plans, Philadelphia: S.I.A.M. (1982) 